\title{
Accessible information without disturbing partially known quantum states on a von Neumann algebra
}

\author{
Yui Kuramochi
}

Received: date / Accepted: date

\begin{abstract}
This paper addresses the problem of how much information we can extract without disturbing a statistical experiment, which is a family of partially known normal states on a von Neumann algebra. We define the classical part of a statistical experiment as the restriction of the equivalent minimal sufficient statistical experiment to the center of the outcome space, which, in the case of density operators on a Hilbert space, corresponds to the classical probability distributions appearing in the maximal decomposition by Koashi and Imoto [Phys. Rev. A 66, 022318 (2002)]. We show that we can access by a Schwarz or completely positive channel at most the classical part of a statistical experiment if we do not disturb the states. We apply this result to the broadcasting problem of a statistical experiment. We also show that the classical part of the direct product of statistical experiments is the direct product of the classical parts of the statistical experiments. The proof of the latter result is based on the theorem that the direct product of minimal sufficient statistical experiments is also minimal sufficient.
\end{abstract}

Keywords minimal sufficiency · tensor products of operator algebras · classical part of statistical experiment - direct product of statistical experiments

Mathematics Subject Classification (2010) 81P45 - 46L53 - 62B15 · 47L90

\section{Introduction}

One of the fundamental feature of quantum theory is the impossibility of extracting information without disturbing unknown quantum states. We can see this characteristic, for example, from the no-cloning theorem [18,24,6] or more general no-

This work was supported by the National Natural Science Foundation of China (Grants No. 11374375 and No. 11574405).

Yui Kuramochi

School of Physics and Astronomy, Sun Yat-Sen University (Zhuhai Campus), Zhuhai 519082, China

E-mail: yui.tasuke.kuramochi@gmail.com 
broadcasting theorem [2,1], which states that cloning or broadcasting operation can be realized only for restricted family of quantum states.

Recently, the cloning and broadcasting conditions were considered in general operator algebraic framework [10,11], in which the mean ergodic theorem for von Neumann algebras [13] plays a fundamental role. With the help of the mean ergodic theorem, we can also establish the existence of a minimal sufficient statistical experiment equivalent to a given operator algebraic statistical experiment [14]. If a statistical experiment is a family of density operators on a Hilbert space, the equivalent minimal sufficient statistical experiment corresponds to the maximal decomposition by Koashi and Imoto [12].

We can regard the broadcasting as a special class of operations that do not disturb a family of partially known states. A typical example of such an operation other than the broadcasting is as follows: if a family of density operators $\left(\rho_{\theta}\right)_{\theta \in \Theta}$ commutes with a complete set of projections $\left(P_{j}\right)$, the corresponding projective measurement does not disturb the states $\left(\rho_{\theta}\right)_{\theta \in \Theta}$.

This motivates us to ask the following question: how much information can we extract without disturbing a family of partially known quantum states? The present paper addresses this problem in the von Neumann algebra framework. We show that if we do not disturb a statistical experiment, we can access at most what we call the classical part of the statistical experiment, even if we allow non-classical outcome spaces. We mention that this problem was also considered in [12] for finite-dimensional density operators.

This paper is organized as follows. After mathematical preliminaries in Section 2 we introduce in Section 3 the classical part of a statistical experiment, which is defined as the restriction of the equivalent minimal sufficient statistical experiment to the center of the outcome space (Definition 2). We show that we can access at most the classical part by a Schwarz channel without disturbing a given statistical experiment (Theorem 1). From this, the no-broadcasting theorem immediately follows (Section 3.2. Corollary 1). In Section 3.3 we consider the case of density operators on a (possibly infinite-dimensional, or even non-separable) Hilbert space and find that the classical part in this case corresponds to the classical probability distributions appearing in the maximal decomposition in [12]. In Section 4] we show that the classical part of the direct product of two statistical experiments coincides with the direct product of the classical parts of the statistical experiments (Theorem 3). The proof of Theorem 3 is based on Theorem 2 which states that the direct product of two minimal sufficient statistical experiments is also minimal sufficient. Finally, Section 5 concludes the paper.

\section{Preliminaries}

In this section, we introduce mathematical preliminaries on operator algebras, channels between them, and (minimal sufficient) statistical experiment. For a general reference of operator algebras, we refer [20]. 
2.1 States and channels on operator algebras.

Throughout this paper, we only consider $*-, C^{*}$-, and von Neumann algebras with unit elements in the multiplications. The unit element of a $*$-algebra $\mathscr{A}$ is denoted by $\mathbb{1}_{\mathscr{A}}$. We denote by $\operatorname{id}_{\mathscr{A}}$ the identity map on a $*$-algebra $\mathscr{A}$ and by $\mathbb{M}_{n}(\mathscr{A})$ the set of $n \times n$ matrices with entries from $\mathscr{A}$. We denote by $\mathfrak{Z}(\mathscr{A})$ the center $\{Z \in \mathscr{A} \mid A Z=Z A(\forall A \in \mathscr{A})\}$ of $\mathscr{A}$. The algebra of bounded operators on a Hilbert space $\mathscr{H}$ is denoted by $\mathscr{L}(\mathscr{H})$.

A positive linear functional on a $C^{*}$-algebra $\mathscr{A}$ satisfying the normalization condition $\varphi\left(\mathbb{1}_{\mathscr{A}}\right)=1$ is called a state on $\mathscr{A}$. A state $\varphi$ on a von Neumann algebra $\mathscr{M}$ is called normal if $\varphi\left(\sup _{i} A_{i}\right)=\sup _{i} \varphi\left(A_{i}\right)$ for any monotonically increasing bounded net $\left(A_{i}\right)$ on $\mathscr{M}$. A state on $\mathscr{M}$ is normal if and only if $\varphi$ is continuous in the ultraweak topology on $\mathscr{M}$. The sets of ultraweakly continuous linear functionals and normal states on $\mathscr{M}$ are denoted by $\mathscr{M}_{*}$ and $\mathscr{S}_{\sigma}(\mathscr{M})$, respectively. The support $\mathrm{s}(\varphi)$ of $\varphi \in \mathscr{S}_{\sigma}(\mathscr{M})$ is the minimal projection $\mathrm{s}(\varphi) \in \mathscr{M}$ satisfying $\varphi(\mathrm{s}(\varphi))=1$. For each $\varphi \in \mathscr{S}_{\sigma}(\mathscr{M})$ and each $A \in \mathscr{M}$, we have

$$
\varphi(A)=\varphi(\mathrm{s}(\varphi) A \mathrm{~s}(\varphi))
$$

For positive $A \in \mathscr{M}, \varphi(A)=0$ implies $\mathrm{s}(\varphi) A \mathrm{~s}(\varphi)=0$.

Let $\mathscr{A}$ be a $C^{*}$-algebra and let $\pi_{\mathscr{A}}$ be the universal representation of $\mathscr{A}$ acting on the Hilbert space $\mathscr{H}_{\mathscr{A}}$, i.e. $\pi_{\mathscr{A}}: \mathscr{A} \rightarrow \mathscr{L}\left(\mathscr{H}_{\mathscr{A}}\right)$ is the direct sum of the GNS representations taken over all the states on $\mathscr{A}$. The von Neumann algebra $\pi_{\mathscr{A}}(\mathscr{A})^{\prime \prime}$, where the prime denotes the commutant, is called the enveloping von Neumann algebra of $\mathscr{A}$ and denoted by $\mathscr{A}^{* *}$. The enveloping von Neumann algebra $\mathscr{A}^{* *}$ is, as a Banach space, isometrically isomorphic to the double dual of $\mathscr{A}$. Every linear functional $\phi \in \mathscr{A}^{*}$ extends to a linear functional $\bar{\phi} \in\left(\mathscr{A}^{* *}\right)_{*}$, i.e. $\bar{\phi}$ is the ultraweakly continuous linear functional on $\mathscr{A}^{* *}$ satisfying $\phi=\bar{\phi} \circ \pi_{\mathscr{A}}$. The enveloping von Neumann algebra satisfies the following universal property: for each representation $\pi: \mathscr{A} \rightarrow \mathscr{L}(\mathscr{K})$ acting on the Hilbert space $\mathscr{K}$, there exists a normal representation $\bar{\pi}: \mathscr{A}^{* *} \rightarrow \mathscr{L}(\mathscr{K})$ that is an extension of $\pi$, i.e. $\pi=\bar{\pi} \circ \pi_{\mathscr{A}}$. If there is no confusion, we identify $\mathscr{A}$ with the $C^{*}$-subalgebra $\pi_{\mathscr{A}}(\mathscr{A})$ of $\mathscr{A}^{* *}$.

Next, we introduce channels. In this paper, we consider channels whose outcome spaces are algebraic tensor products of von Neumann algebras and do not in general have unique $C^{*}$-norms. For this reason, we slightly generalize the notion of channels as follows. Let $\mathscr{A}$ be a $*$-algebra, let $\mathscr{B}$ be a $C^{*}$-algebra, and let $\Lambda: \mathscr{A} \rightarrow \mathscr{B}$ a linear map. $\Lambda$ is called unital if $\Lambda\left(\mathbb{1}_{\mathscr{A}}\right)=\mathbb{1}_{\mathscr{B}}$. For an integer $n \geq 1, \Lambda$ is called $n$-positive if

$$
\sum_{i, j=1}^{n} B_{i}^{*} \Lambda\left(A_{i}^{*} A_{j}\right) B_{j} \geq 0
$$

for each $A_{i} \in \mathscr{A}$ and each $B_{i} \in \mathscr{B}(i=1, \ldots, n) . \Lambda$ is called completely positive (CP) if $\Lambda$ is $n$-positive for every positive integer $n$. A unital map $\Lambda: \mathscr{A} \rightarrow \mathscr{B}$ is called Schwarz if $\Lambda$ satisfies

$$
\begin{gathered}
\Lambda\left(A^{*}\right)=\Lambda(A)^{*}, \\
\Lambda\left(A^{*} A\right) \geq \Lambda\left(A^{*}\right) \Lambda(A)
\end{gathered}
$$


for all $A \in \mathscr{A}$. The Schwarz condition is equivalent to the following single matrix inequality:

$$
\left(\begin{array}{cc}
\Lambda\left(A^{*} A\right) & \Lambda\left(A^{*}\right) \\
\Lambda(A) & \mathbb{1}_{\mathscr{B}}
\end{array}\right) \geq 0
$$

A unital Schwarz map is positive, i.e. 1-positive. A unital positive map $\Lambda: \mathscr{A} \rightarrow \mathscr{B}$ is called a channel (in the Heisenberg picture). The codomain $\mathscr{B}$ and domain $\mathscr{A}$ of a channel $\Lambda: \mathscr{A} \rightarrow \mathscr{B}$ are called the input and outcome spaces, or algebras, of $\Lambda$, respectively. A channel $\Lambda: \mathscr{A} \rightarrow \mathscr{B}$ maps a state $\phi$ on the input space $\mathscr{B}$ to the normalized positive linear functional $\phi \circ \Lambda$ on $\mathscr{A}$. The sets of CP and Schwarz channels from $\mathscr{A}$ to $\mathscr{B}$ are denoted by $\mathbf{C h}{ }^{\mathrm{CP}}(\mathscr{A} \rightarrow \mathscr{B})$ and $\mathbf{C h}^{\mathrm{Sch}}(\mathscr{A} \rightarrow \mathscr{B})$, respectively. As in the case of channels with outcome $C^{*}$-algebras [5], we can show that any 2-positive (and therefore any $\mathrm{CP}$ ) channel $\Lambda: \mathscr{A} \rightarrow \mathscr{B}$ is a Schwarz channel. If either $\mathscr{A}$ or $\mathscr{B}$ is a commutative $C^{*}$-algebra, a linear map $\Lambda: \mathscr{A} \rightarrow \mathscr{B}$ is CP if and only if $\Lambda$ is positive.

Let $\Lambda \in \mathbf{C h}^{\operatorname{Sch}}(\mathscr{A} \rightarrow \mathscr{B})$ be a Schwarz channel with input $C^{*}$-algebra $\mathscr{B}$ and outcome $*$-algebra $\mathscr{A}$. We define the multiplicative domain of $\Lambda$ by

$$
\mathscr{M}_{\Lambda}:=\left\{A \in \mathscr{A} \mid \Lambda\left(A^{*} A\right)=\Lambda\left(A^{*}\right) \Lambda(A), \Lambda\left(A A^{*}\right)=\Lambda(A) \Lambda\left(A^{*}\right)\right\} .
$$

The following lemma for the multiplicative domain of a Schwarz channel can be shown analogously as in [8] (Lemma 3.9).

Lemma 1 Let $\mathscr{A}$ be a $*$-algebra, let $\mathscr{B}$ be a $C^{*}$-algebra, and let $\Lambda \in \mathbf{C h}^{\mathrm{Sch}}(\mathscr{A} \rightarrow \mathscr{B})$ be a Schwarz channel. Then for $A \in \mathscr{A}, A \in \mathscr{M}_{\Lambda}$ if and only if $\Lambda(B A)=\Lambda(B) \Lambda(A)$ and $\Lambda(A B)=\Lambda(A) \Lambda(B)$ for all $B \in \mathscr{A}$.

From Lemma 1 the multiplicative domain $\mathscr{M}_{\Lambda}$ is a unital $*$-subalgebra of $\mathscr{A}$.

Let $\mathscr{M}$ and $\mathscr{N}$ be von Neumann algebras. A channel $\Lambda: \mathscr{M} \rightarrow \mathscr{N}$ is called normal if it is continuous in the ultraweak topologies of $\mathscr{M}$ and $\mathscr{N}$, respectively. The set of normal CP (respectively, Schwarz) channels from $\mathscr{M}$ to $\mathscr{N}$ is denoted by $\mathbf{C h}_{\sigma}^{\mathrm{CP}}(\mathscr{M} \rightarrow \mathscr{N})$ (respectively, $\mathbf{C h}_{\sigma}^{\mathrm{Sch}}(\mathscr{M} \rightarrow \mathscr{N})$ ). We also write $\mathbf{C h}_{\sigma}^{\mathrm{CP}}(\mathscr{M}):=$ $\mathbf{C h}_{\sigma}^{\mathrm{CP}}(\mathscr{M} \rightarrow \mathscr{M})$ and $\mathbf{C h}_{\sigma}^{\mathrm{Sch}}(\mathscr{M}):=\mathbf{C h}_{\sigma}^{\mathrm{Sch}}(\mathscr{M} \rightarrow \mathscr{M})$.

\subsection{Tensor products of operator algebras}

Let $\mathscr{A}$ and $\mathscr{B}$ be $C^{*}$-algebras. We denote by $\mathscr{A} \otimes_{\text {alg }} \mathscr{B}$ the algebraic tensor product of $\mathscr{A}$ and $\mathscr{B}$. The structure of a $*$-algebra is naturally induced to the algebraic tensor product $\mathscr{A} \otimes_{\text {alg }} \mathscr{B}$ by

$$
\begin{gathered}
\left(\sum_{i} A_{i} \otimes B_{i}\right)\left(\sum_{j} C_{j} \otimes D_{j}\right):=\sum_{i, j} A_{i} C_{j} \otimes B_{i} D_{j}, \\
\left(\sum_{i} A_{i} \otimes B_{i}\right)^{*}:=\sum_{i} A_{i}^{*} \otimes B_{i}^{*}
\end{gathered}
$$

$\left(A_{i}, C_{j} \in \mathscr{A} ; B_{i}, D_{j} \in \mathscr{B}\right)$. A norm $\|\cdot\|_{\gamma}$ on $\mathscr{A} \otimes_{\text {alg }} \mathscr{B}$ satisfying $\|X Y\|_{\gamma} \leq\|X\|_{\gamma}\|Y\|_{\gamma}$, $\left\|X^{*}\right\|_{\gamma}=\|X\|_{\gamma}$, and $\left\|X^{*} X\right\|_{\gamma}=\|X\|_{\gamma}^{2}\left(X, Y \in \mathscr{A} \otimes_{\text {alg }} \mathscr{B}\right)$ is called a $C^{*}$-norm on 
$\mathscr{A} \otimes_{\text {alg }} \mathscr{B}$. The completion of $\mathscr{A} \otimes_{\text {alg }} \mathscr{B}$ with respect to the $C^{*}$-norm $\|\cdot\|_{\gamma}$, written as $\mathscr{A} \otimes_{\gamma} \mathscr{B}$, is a $C^{*}$-algebra and called a $C^{*}$-tensor product of $\mathscr{A}$ and $\mathscr{B}$. We denote the injective norm, the injective tensor product, the projective norm, and the projective tensor product by $\|\cdot\|_{\min }, \mathscr{A} \otimes_{\min } \mathscr{B},\|\cdot\|_{\max }$, and $\mathscr{A} \otimes_{\max } \mathscr{B}$, respectively. We have $\|X\|_{\min } \leq\|X\|_{\gamma} \leq\|X\|_{\max }\left(X \in \mathscr{A} \otimes_{\text {alg }} \mathscr{B}\right)$ for any $C^{*}$-norm $\|\cdot\|_{\gamma}$ on $\mathscr{A} \otimes_{\text {alg }} \mathscr{B}$. If either $\mathscr{A}$ or $\mathscr{B}$ is commutative, then we have $\|\cdot\|_{\min }=\|\cdot\|_{\max }$ and the $C^{*}$-norm is unique in this case.

Let $\mathscr{M}$ and $\mathscr{N}$ be von Neumann algebras acting on Hilbert spaces $\mathscr{H}$ and $\mathscr{K}$, respectively. The von Neumann algebra on $\mathscr{H} \otimes \mathscr{K}$ generated by $\{A \otimes B \mid A \in \mathscr{M}, B \in$ $\mathscr{N}\}$ is called the normal tensor product of $\mathscr{M}$ and $\mathscr{N}$, denoted by $\mathscr{M} \bar{\otimes} \mathscr{N}$.

Let $\mathscr{M}$ and $\mathscr{N}$ be von Neumann algebras, let $\mathscr{B}$ be a $C^{*}$-algebra, and let $\mathscr{M} \otimes_{y} \mathscr{B}$ be the algebraic or a $C^{*}$-tensor product of $\mathscr{M}$ and $\mathscr{B}$. A linear map $\Lambda: \mathscr{M} \otimes_{y} \mathscr{B} \rightarrow \mathscr{N}$ is called left-normal if the linear map

$$
\Lambda_{B}: \mathscr{M} \ni A \mapsto \Lambda(A \otimes B) \in \mathscr{N}
$$

is ultraweakly continuous for each $B \in \mathscr{B}$.

Let $\mathscr{M}_{1}, \mathscr{M}_{2}$, and $\mathscr{N}$ be von Neumann algebras and let $\mathscr{M}_{1} \otimes_{y} \mathscr{M}_{2}$ be the algebraic or a $C^{*}$-tensor product of $\mathscr{M}_{1}$ and $\mathscr{M}_{2}$. A linear map $\mathscr{M}_{1} \otimes_{y} \mathscr{M}_{2}: \rightarrow \mathscr{N}$ is called binormal if the linear maps

$$
\begin{gathered}
\Lambda_{A_{2}^{\prime}}^{L}: \mathscr{M}_{1} \ni A_{1} \mapsto \Lambda\left(A_{1} \otimes A_{2}^{\prime}\right) \in \mathscr{N}, \\
\Lambda_{A_{1}^{\prime}}^{R}: \mathscr{M}_{2} \ni A_{2} \mapsto \Lambda\left(A_{1}^{\prime} \otimes A_{2}\right) \in \mathscr{N}
\end{gathered}
$$

are ultraweakly continuous for each $A_{1}^{\prime} \in \mathscr{M}_{1}$ and each $A_{2}^{\prime} \in \mathscr{M}_{2}$.

Proposition 1 ([20], Propositions IV.4.23 and IV.5.13) Let $\mathscr{A}, \mathscr{B}, \mathscr{C}$, and $\mathscr{D}$ be $C^{*}$. algebras and let $\Lambda \in \mathbf{C h}^{\mathrm{CP}}(\mathscr{A} \rightarrow \mathscr{B})$ and $\Gamma \in \mathbf{C h}^{\mathrm{CP}}(\mathscr{C} \rightarrow \mathscr{D})$ be CP channels. Define the algebraic tensor product map $\Lambda \otimes_{\mathrm{alg}} \Gamma: \mathscr{A} \otimes_{\mathrm{alg}} \mathscr{C} \rightarrow \mathscr{B} \otimes_{\mathrm{alg}} \mathscr{D}$ by

$$
\Lambda \otimes_{\mathrm{alg}} \Gamma\left(\sum_{i} A_{i} \otimes C_{i}\right):=\sum_{i} \Lambda\left(A_{i}\right) \otimes \Gamma\left(B_{i}\right)
$$

$\left(A_{i} \in \mathscr{A}, C_{i} \in \mathscr{C}\right)$. Then $\Lambda \otimes_{\mathrm{alg}} \Gamma$ uniquely extends to a $C P$ channel $\Lambda \otimes_{\min } \Gamma \in$ $\mathbf{C h}^{\mathrm{CP}}\left(\mathscr{A} \otimes_{\min } \mathscr{C} \rightarrow \mathscr{B} \otimes_{\min } \mathscr{D}\right)$. If we further assume that $\mathscr{A}, \mathscr{B}, \mathscr{C}$, and $\mathscr{D}$ are von Neumann algebras and that $\Lambda$ and $\Gamma$ are normal, then $\Lambda \otimes_{\text {alg }} \Gamma$ uniquely extends to a normal channel $\Lambda \bar{\otimes} \Gamma \in \mathbf{C h}_{\sigma}^{\mathrm{CP}}(\mathscr{A} \bar{\otimes} \mathscr{C} \rightarrow \mathscr{B} \bar{\otimes} \mathscr{D})$.

A special case of Proposition 1 is normal states on von Neumann algebras: if $\varphi$ and $\psi$ are normal states on von Neumann algebras $\mathscr{M}$ and $\mathscr{N}$, respectively, then the linear functional $\varphi \otimes_{\text {alg }} \psi$ on $\mathscr{M} \otimes_{\text {alg }} \mathscr{N}$ uniquely extends to a normal state $\varphi \bar{\otimes} \psi$ on $\mathscr{M} \bar{\otimes} \mathscr{N}$ 
2.3 Coarse-graining relations for statistical experiments

A statistical experiment is a triple $\mathscr{E}=\left(\mathscr{M}, \Theta,\left(\varphi_{\theta}\right)_{\theta \in \Theta}\right)$ such that $\mathscr{M}$ is a von Neumann algebra, $\Theta$ is a set, and $\left(\varphi_{\theta}\right)_{\theta \in \Theta} \in \mathscr{S}_{\sigma}(\mathscr{M})^{\Theta}$ is a family of normal states on $\mathscr{M}$ indexed by $\Theta . \mathscr{M}$ and $\Theta$ are called the outcome space and the parameter set of $\mathscr{E}$, respectively. Operationally, a statistical experiment $\mathscr{E}=\left(\mathscr{M}, \Theta,\left(\varphi_{\theta}\right)_{\theta \in \Theta}\right)$ corresponds to the situation in which the state on the outcome space $\mathscr{M}$ is known to be one of the states $\left(\varphi_{\theta}\right)_{\theta \in \Theta}$. The family of normal states $\left(\varphi_{\theta}\right)_{\theta \in \Theta}$ is called faithful if for any positive $A \in \mathscr{M}, \varphi_{\theta}(A)=0$ for all $\theta \in \Theta$ implies $A=0$. If so, we also say that $\mathscr{E}$ is faithful. $\mathscr{E}$ is faithful if and only if

$$
\bigvee_{\theta \in \Theta} \mathrm{s}\left(\varphi_{\theta}\right)=\mathbb{1}_{\mathscr{M}}
$$

A statistical experiment $\mathscr{E}$ is called classical if the outcome space of $\mathscr{E}$ is commutative.

For a statistical experiment $\mathscr{E}=\left(\mathscr{M}, \Theta,\left(\varphi_{\theta}\right)_{\theta \in \Theta}\right)$ we define the normal extension of $\mathscr{E}$ by $\overline{\mathscr{E}}:=\left(\mathscr{M}^{* *}, \Theta,\left(\overline{\varphi_{\theta}}\right)_{\theta \in \Theta}\right)$, where $\overline{\varphi_{\theta}}$ is the normal extension of $\varphi_{\theta}$ to $\mathscr{M}^{* *}$.

Let $\mathscr{E}=\left(\mathscr{M}, \Theta,\left(\varphi_{\theta}\right)_{\theta \in \Theta}\right)$ and $\mathscr{F}=\left(\mathscr{N}, \Theta,\left(\psi_{\theta}\right)_{\theta \in \Theta}\right)$ be statistical experiments with a common parameter set $\Theta$. We introduce the following coarse-graining (or randomization) and isomorphism relations for statistical experiments:

- $\mathscr{E} \preccurlyeq \mathrm{CP} \mathscr{F}(\mathscr{E}$ is a coarse-graining of $\mathscr{F}): \stackrel{\text { def. }}{\Leftrightarrow} \exists \Phi \in \mathbf{C h}^{\mathrm{CP}}(\mathscr{M} \rightarrow \mathscr{N})$ s.t. $\left[\varphi_{\theta}=\right.$ $\left.\psi_{\theta} \circ \Phi(\forall \theta \in \Theta)\right]$

- $\mathscr{E} \preccurlyeq \mathrm{CP}_{\sigma} \mathscr{F}(\mathscr{E}$ is a normal coarse-graining of $\mathscr{F}): \stackrel{\text { def. }}{\Leftrightarrow} \exists \Phi \in \mathbf{C h}_{\sigma}^{\mathrm{CP}}(\mathscr{M} \rightarrow \mathscr{N})$ s.t. $\left[\varphi_{\theta}=\psi_{\theta} \circ \Phi(\forall \theta \in \Theta)\right]$;

- $\mathscr{E} \sim \mathrm{CP} \mathscr{F}(\mathscr{E}$ is $\mathrm{CP}$ equivalent to $\mathscr{F})$ : def. $\mathscr{E} \preccurlyeq \mathrm{CP} \mathscr{F}$ and $\mathscr{F} \preccurlyeq \mathrm{CP} \mathscr{E}$;

- $\mathscr{E} \sim \mathrm{CP}_{\sigma} \mathscr{F}(\mathscr{E}$ is normally CP equivalent to $\mathscr{F}): \stackrel{\text { def. }}{\Leftrightarrow} \mathscr{E} \preccurlyeq \mathrm{CP}_{\sigma} \mathscr{F}$ and $\mathscr{F} \preccurlyeq \mathrm{CP}_{\sigma} \mathscr{E}$;

- $\mathscr{E} \cong \mathscr{F}$ ( $\mathscr{E}$ and $\mathscr{F}$ are normally isomorphic) $: \stackrel{\text { def. }}{\Leftrightarrow}$ there exists a normal isomorphism $\pi: \mathscr{M} \rightarrow \mathscr{N}$ such that $\varphi_{\theta}=\psi_{\theta} \circ \pi$ for all $\theta \in \Theta$.

The relations $\preccurlyeq \mathrm{CP}$ and $\preccurlyeq \mathrm{CP}_{\sigma}$ are binary preorder relations for statistical experiments, and $\sim_{\mathrm{CP}}, \sim_{\mathrm{CP}_{\sigma}}$, and $\cong$ are binary equivalence relations.

The following two lemmas, which will be used in the proof of Theorem 1, are due to [15] (Corollaries 3 and 4).

Lemma 2 Let $\mathscr{E}=\left(\mathscr{M}, \Theta,\left(\varphi_{\theta}\right)_{\theta \in \Theta}\right)$ be a statistical experiment and let $\overline{\mathscr{E}}=\left(\mathscr{M}^{* *}, \Theta,\left(\overline{\varphi_{\theta}}\right)_{\theta \in \Theta}\right)$ be the normal extension of $\mathscr{E}$. Then $\mathscr{E} \sim \mathrm{CP}_{\sigma} \overline{\mathscr{E}}$.

Lemma 3 Let $\mathscr{E}=\left(\mathscr{M}, \Theta,\left(\varphi_{\theta}\right)_{\theta \in \Theta}\right)$ and $\mathscr{F}=\left(\mathscr{N}, \Theta,\left(\psi_{\theta}\right)_{\theta \in \Theta}\right)$ be statistical experiments with a common parameter set $\Theta$. Then $\mathscr{E} \preccurlyeq \mathrm{CP} \mathscr{F}$ (respectively, $\mathscr{E} \sim{ }_{\mathrm{CP}} \mathscr{F}$ ) if and only if $\mathscr{E} \preccurlyeq \mathrm{CP}_{\sigma} \mathscr{F}$ (respectively, $\mathscr{E} \sim \mathrm{CP}_{\sigma} \mathscr{F}$ ).

The proof in [15] is based on the results on the compatibility relations of CP channels and requires a number of irrelevant discussions. For readers' convenience, we give other direct proofs of these lemmas in Appendix A 
2.4 Minimal sufficient subalgebra and statistical experiment

Let $\mathscr{A}$ be a $C^{*}$-algebra and let $\mathscr{B}$ be a $C^{*}$-subalgebra of $\mathscr{A}$. A linear map $\mathbb{E}: \mathscr{A} \rightarrow \mathscr{B}$ is called a conditional expectation onto $\mathscr{B}$ if $\mathbb{E}(B)=B$ for all $B \in \mathscr{B}$ and $\|\mathbb{E}(A)\| \leq$ $\|A\|$ for all $A \in \mathscr{A}$. Tomiyama's theorem (e.g. [3], Theorem 1.5.10) states that a conditional expectation $\mathbb{E}$ onto $\mathscr{B}$ satisfies $\mathbb{E}\left(B_{1} A B_{2}\right)=B_{1} \mathbb{E}(A) B_{2}\left(A \in \mathscr{A} ; B_{1}, B_{2} \in \mathscr{B}\right)$. From this we also have $\mathbb{E} \in \mathbf{C h}^{\mathrm{CP}}(\mathscr{A} \rightarrow \mathscr{B})$.

Let $\mathscr{E}=\left(\mathscr{M}, \Theta,\left(\varphi_{\theta}\right)_{\theta \in \Theta}\right)$ be a statistical experiment and let $\mathscr{N} \subseteq \mathscr{M}$ be a von Neumann subalgebra of $\mathscr{M} . \mathscr{N}$ is said to be sufficient (in the sense of Umegaki [22, 23,17]) with respect to the family $\left(\varphi_{\theta}\right)_{\theta \in \Theta}$ if there exists a normal conditional expectation $\mathbb{E}$ from $\mathscr{M}$ onto $\mathscr{N}$ such that $\varphi_{\theta} \circ \mathbb{E}=\varphi_{\theta}$ for all $\theta \in \Theta . \mathscr{N}$ is said to be a minimal sufficient subalgebra with respect to $\left(\varphi_{\theta}\right)_{\theta \in \Theta}$ if $\mathscr{N}$ is sufficient and contained in any sufficient subalgebra. A minimal sufficient subalgebra is, if exists, unique for a given family of normal states.

A statistical experiment $\mathscr{E}=\left(\mathscr{M}, \Theta,\left(\varphi_{\theta}\right)_{\theta \in \Theta}\right)$ is called minimal sufficient if $\mathscr{E}$ satisfies either of the following equivalent conditions ([14], Theorem 2):

(i) for $\Phi \in \mathbf{C h}_{\sigma}^{\mathrm{CP}}(\mathscr{M}), \varphi_{\theta} \circ \Phi=\varphi_{\theta}$ for all $\theta \in \Theta$ implies $\Phi=\mathrm{id} \mathscr{M}$;

(ii) for $\Phi \in \mathbf{C h}_{\sigma}^{\text {Sch }}(\mathscr{M}), \varphi_{\theta} \circ \Phi=\varphi_{\theta}$ for all $\theta \in \Theta$ implies $\Phi=\mathrm{id}_{\mathscr{M}}$;

(iii) $\left(\varphi_{\theta}\right)_{\theta \in \Theta}$ is faithful and $\mathscr{M}$ is a minimal sufficient subalgebra with respect to $\left(\varphi_{\theta}\right)_{\theta \in \Theta}$.

From condition (iii), a minimal sufficient statistical experiment is faithful.

Let $\mathscr{E}=\left(\mathscr{M}, \Theta,\left(\varphi_{\theta}\right)_{\theta \in \Theta}\right)$ be an arbitrary statistical experiment. It is known that there exists a minimal sufficient statistical experiment $\mathscr{E}_{0}$ satisfying $\mathscr{E} \sim \mathrm{CP}_{\sigma} \mathscr{E} 0$ ([14], Theorem 1). Such a minimal sufficient statistical experiment $\mathscr{E}_{0}$ is unique up to normal isomorphism and, in this sense, we may say that $\mathscr{E}_{0}$ is the minimal sufficient statistical experiment normally CP equivalent to $\mathscr{E}$. If $\mathscr{E}$ is faithful, $\mathscr{E}_{0}$ can be constructed as follows [14,17]. Define

$$
\begin{gathered}
\mathbb{F}:=\left\{\Phi \in \mathbf{C h}_{\sigma}^{\mathrm{CP}}(\mathscr{M}) \mid \varphi_{\theta} \circ \Phi=\varphi_{\theta}(\forall \theta \in \Theta)\right\} \\
\mathscr{M}_{0}:=\{A \in \mathscr{M} \mid \Phi(A)=A(\forall \Phi \in \mathbb{F})\} .
\end{gathered}
$$

Then from the mean ergodic theorem for von Neumann algebras [13], there exists a normal conditional expectation $\mathbb{E}$ from $\mathscr{M}$ onto $\mathscr{M}_{0}$ such that

$$
\begin{gathered}
\varphi_{\theta} \circ \mathbb{E}=\varphi_{\theta} \quad(\forall \theta \in \Theta), \\
\mathbb{E} \circ \Phi=\Phi \circ \mathbb{E}=\mathbb{E} \quad(\forall \Phi \in \mathbb{F}) .
\end{gathered}
$$

If we write as $\varphi_{\theta}^{(0)}$ the restriction of $\varphi_{\theta}$ to $\mathscr{M}_{0}$, then we can show that $\mathscr{E}_{0}:=\left(\mathscr{M}_{0}, \Theta,\left(\varphi_{\theta}^{(0)}\right)_{\theta \in \Theta}\right)$ is the desired minimal sufficient statistical experiment. In this case, $\mathscr{M}_{0}$ is the minimal sufficient subalgebra with respect to $\left(\varphi_{\theta}\right)_{\theta \in \Theta}$. We note that the condition (1) for a conditional expectation $\mathbb{E}$ onto $\mathscr{M}_{0}$ uniquely determines $\mathbb{E}$; indeed if $\mathbb{E}^{\prime}$ is a normal conditional expectation from $\mathscr{M}$ onto $\mathscr{M}_{0}$ satisfying $\varphi_{\theta} \circ \mathbb{E}^{\prime}=\varphi_{\theta}$ for all $\theta \in \Theta$, then condition (2) implies $\mathbb{E}^{\prime}=\mathbb{E} \circ \mathbb{E}^{\prime}=\mathbb{E}$. 


\section{Accessible part of statistical experiment}

In this section, we prove the main result (Theorem 1) and apply it to the no-broadcasting theorem and the case of density operators on a Hilbert space.

\subsection{Accessible and classical parts of a statistical experiment}

We first define the accessibility relations for statistical experiments as follows.

Definition 1 (accessibility relations for statistical experiments) Let $\mathscr{E}=\left(\mathscr{M}, \Theta,\left(\varphi_{\theta}\right)_{\theta \in \Theta}\right)$ and $\mathscr{F}=\left(\mathscr{N}, \Theta,\left(\psi_{\theta}\right)_{\theta \in \Theta}\right)$ be statistical experiments with a common parameter set $\Theta$, let $X$ be either CP or Sch, and let $\mathscr{M} \otimes_{y} \mathscr{N}$ be the algebraic or a $C^{*}$-tensor product of $\mathscr{M}$ and $\mathscr{N}$.

1. A channel $\Lambda \in \mathbf{C h}^{X}\left(\mathscr{M} \otimes_{y} \mathscr{N} \rightarrow \mathscr{M}\right)$ is said to extract $\mathscr{F}$ without disturbing $\mathscr{E}$ if for each $\theta \in \Theta$, each $A \in \mathscr{M}$, and each $B \in \mathscr{N}$, it holds that

$$
\begin{aligned}
& \varphi_{\theta}(A)=\varphi_{\theta} \circ \Lambda\left(A \otimes \mathbb{1}_{\mathscr{N}}\right), \\
& \psi_{\theta}(B)=\varphi_{\theta} \circ \Lambda\left(\mathbb{1}_{\mathscr{M}} \otimes B\right) .
\end{aligned}
$$

2. $\mathscr{F}$ is said to be accessible without disturbing $\mathscr{E}$ in the sense of $X$ channel and the tensor product $\otimes_{y}$, written as $\mathscr{F} \Subset_{y}^{X} \mathscr{E}$, if there exists a channel $\Lambda \in \mathbf{C h}^{X}\left(\mathscr{M} \otimes_{y}\right.$ $\mathscr{N} \rightarrow \mathscr{M})$ that extracts $\mathscr{F}$ without disturbing $\mathscr{E}$.

3. We write $\mathscr{F} \Subset_{y, \text { bin }}^{X} \mathscr{E}$ (respectively, $\mathscr{F} \Subset_{y, \text { lnor }}^{X} \mathscr{E}$ ) if there exists a binormal (respectively, left-normal) channel $\Lambda \in \mathbf{C h}^{X}\left(\mathscr{M} \otimes_{y} \mathscr{N} \rightarrow \mathscr{M}\right)$ that extracts $\mathscr{F}$ without disturbing $\mathscr{E}$.

Let us consider a special case in which $\mathscr{M}$ and $\mathscr{N}$ are the full operator algebras $\mathscr{L}(\mathscr{H})$ and $\mathscr{L}(\mathscr{K})$ on finite-dimensional Hilbert spaces $\mathscr{H}$ and $\mathscr{K}$, respectively. As usual, we identify the normal states $\varphi_{\theta}$ and $\psi_{\theta}$ with density operators $\rho_{\theta}$ on $\mathscr{H}$ and $\sigma_{\theta}$ on $\mathscr{K}$, respectively, such that $\varphi_{\theta}(A)=\operatorname{tr}\left(\rho_{\theta} A\right)$ and $\psi_{\theta}(B)=\operatorname{tr}\left(\sigma_{\theta} B\right)$ $(A \in \mathscr{L}(\mathscr{H}), B \in \mathscr{L}(\mathscr{K}))$. Then a CP channel $\Lambda \in \mathbf{C h}^{\mathrm{CP}}(\mathscr{L}(\mathscr{H} \otimes \mathscr{K}) \rightarrow \mathscr{L}(\mathscr{H}))$ extracts $\mathscr{F}$ without disturbing $\mathscr{E}$ if and only if

$$
\rho_{\theta}=\operatorname{tr}_{\mathscr{K}} \circ \Lambda^{*}\left(\rho_{\theta}\right), \quad \sigma_{\theta}=\operatorname{tr}_{\mathscr{H}} \circ \Lambda^{*}\left(\rho_{\theta}\right)
$$

for all $\theta \in \Theta$, where $\operatorname{tr}_{\mathscr{H}}$ and $\operatorname{tr}_{\mathscr{K}}$ denote the partial traces over $\mathscr{H}$ and $\mathscr{K}$, respectively. If $\mathscr{F}$ is identical to $\mathscr{E}$, the condition (3) reduces to the broadcastability condition for the states $\left(\rho_{\theta}\right)_{\theta \in \Theta}$ and the channel $\Lambda$.

Next, we introduce the classical part of a statistical experiment.

Definition 2 (classical part of statistical experiment) Let $\mathscr{E}=\left(\mathscr{M}, \Theta,\left(\varphi_{\theta}\right)_{\theta \in \Theta}\right)$ be a statistical experiment and let $\mathscr{E}_{0}=\left(\mathscr{M}_{0}, \Theta,\left(\varphi_{\theta}^{(0)}\right)_{\theta \in \Theta}\right)$ be the minimal sufficient statistical experiment satisfying $\mathscr{E} \sim \sim_{\mathrm{CP}_{\sigma}} \mathscr{E} 0$. We define the classical part of $\mathscr{E}$ as the statistical experiment $\left(\mathfrak{Z}\left(\mathscr{M}_{0}\right), \Theta,\left(\varphi_{\theta}^{\mathrm{cl}}\right)_{\theta \in \Theta}\right)$, where $\varphi_{\theta}^{\mathrm{cl}}$ is the restriction of $\varphi_{\theta}^{(0)}$ to the center $\mathfrak{Z}\left(\mathscr{M}_{0}\right)$. The classical part of $\mathscr{E}$ is denoted by $\mathscr{E}_{\text {cl }}$. 
We note that the classical part $\mathscr{E}_{\mathrm{cl}}$ of a statistical experiment $\mathscr{E}$ is well-defined up to normal isomorphism due to the uniqueness of the minimal sufficient $\mathscr{E}_{0}$.

The following theorem, the main result of this paper, states that we can access at most the classical part of a statistical experiment $\mathscr{E}$ without disturbing $\mathscr{E}$; in other words, the classical part $\mathscr{E}_{\mathrm{cl}}$ is the accessible part of $\mathscr{E}$.

Theorem 1 Let $\mathscr{E}=\left(\mathscr{M}, \Theta,\left(\varphi_{\theta}\right)_{\theta \in \Theta}\right), \mathscr{F}=\left(\mathscr{N}, \Theta,\left(\psi_{\theta}\right)_{\theta \in \Theta}\right), X$, and $\mathscr{M} \otimes_{y} \mathscr{N}$ be the same as in Definition [1] and let $z$ be either bin or lnor or blank. Then the condition $\mathscr{F} \Subset_{y, z}^{X} \mathscr{E}$ does not depend on the choices of $X, y$, and $z$, and is equivalent to $\mathscr{F} \preccurlyeq \mathrm{CP}_{\sigma} \mathscr{E}_{\mathrm{Cl}}$.

To prove Theorem 1 we first show the following four lemmas.

Lemma 4 Let $\mathscr{A}, \mathscr{B}$, and $\mathscr{C}$ be $C^{*}$-algebras and let $\Lambda \in \mathbf{C h}^{\mathrm{Sch}}\left(\mathscr{A} \otimes_{\mathrm{alg}} \mathscr{B} \rightarrow \mathscr{C}\right)$ be a Schwarz channel. Then there exists a left-normal Schwarz channel $\tilde{\Lambda} \in \mathbf{C h}^{\mathrm{Sch}}\left(\mathscr{A}^{* *} \otimes_{\mathrm{alg}}\right.$ $\left.\mathscr{B} \rightarrow \mathscr{C}^{* *}\right)$ that is an extension of $\Lambda$.

Proof For each $A \in \mathscr{A}$ and each $B \in \mathscr{B}$, we have

$$
\begin{aligned}
\|\Lambda(A \otimes B)\| & =\left\|\Lambda(A \otimes B)^{*} \Lambda(A \otimes B)\right\|^{1 / 2} \\
& \leq\left\|\Lambda\left((A \otimes B)^{*}(A \otimes B)\right)\right\|^{1 / 2} \\
& =\left\|\Lambda\left(A^{*} A \otimes B^{*} B\right)\right\|^{1 / 2} \\
& \leq\left\|\Lambda\left(\|A\|^{2} \mathbb{1}_{\mathscr{A}} \otimes B^{*} B\right)\right\|^{1 / 2} \\
& \leq\left\|\Lambda\left(\|A\|^{2} \mathbb{1}_{\mathscr{A}} \otimes\|B\|^{2} \mathbb{1}_{\mathscr{B}}\right)\right\|^{1 / 2} \\
& =\|A\|\|B\| .
\end{aligned}
$$

Therefore, for each $B \in \mathscr{B}$, the linear map

$$
\Lambda_{B}: \mathscr{A} \ni A \mapsto \Lambda(A \otimes B) \in \mathscr{C}
$$

is bounded and hence its double dual map $\Lambda_{B}^{* *}: \mathscr{A}^{* *} \rightarrow \mathscr{C}^{* *}$ is an ultraweakly continuous linear map that is an extension of $\Lambda_{B}$. Since $\mathscr{B} \ni B \mapsto \Lambda_{B}$ is linear with respect to $B$, also is $\mathscr{B} \ni B \mapsto \Lambda_{B}^{* *}$. Thus we can define a linear map $\widetilde{\Lambda}: \mathscr{A}^{* *} \otimes_{\text {alg }} \mathscr{B} \rightarrow \mathscr{C}^{* *}$ by $\widetilde{\Lambda}\left(\sum_{i} A_{i}^{\prime \prime} \otimes B_{i}\right):=\sum_{i} \Lambda_{B_{i}}^{* *}\left(A_{i}^{\prime \prime}\right)\left(A_{i}^{\prime \prime} \in \mathscr{A}^{* *}, B_{i} \in \mathscr{B}\right)$. Then $\widetilde{\Lambda}$ is left-normal and an extension of $\Lambda$. Thus it is sufficient to show the Schwarz condition

$$
\left(\begin{array}{cc}
\widetilde{\Lambda}\left(X^{*} X\right) & \widetilde{\Lambda}\left(X^{*}\right) \\
\widetilde{\Lambda}(X) & \mathbb{1}_{\mathscr{C}}
\end{array}\right) \geq 0
$$

for each $X \in \mathscr{A}^{* *} \otimes_{\mathrm{alg}} \mathscr{B}$. We write $X$ as $X=\sum_{i=1}^{n} A_{i}^{\prime \prime} \otimes B_{i}\left(A_{i}^{\prime \prime} \in \mathscr{A}^{* *}, B_{i} \in \mathscr{B}\right)$. Then, from the Kaplansky density theorem, there exist nets $\left(A_{i \alpha}\right)(i=1, \ldots, n)$ on $\mathscr{A}$ satisfying $\left\|A_{i \alpha}\right\| \leq\left\|A_{i}^{\prime \prime}\right\|$ and $A_{i \alpha} \stackrel{u s *}{\longrightarrow} A_{i}^{\prime \prime}$, where $\stackrel{u s *}{\longrightarrow}$ denotes the convergence in the 
ultrastrong* topology on $\mathscr{H}_{\mathscr{A}}$. If we put $X_{\alpha}:=\sum_{i} A_{i \alpha} \otimes B_{i}$, then

$$
\begin{gathered}
\Lambda\left(X_{\alpha}\right)=\sum_{i=1}^{n} \widetilde{\Lambda}\left(A_{i \alpha} \otimes B_{i}\right) \stackrel{u w}{\longrightarrow} \sum_{i=1}^{n} \widetilde{\Lambda}\left(A_{i}^{\prime \prime} \otimes B_{i}\right)=\widetilde{\Lambda}(X), \\
\Lambda\left(X_{\alpha}^{*}\right) \stackrel{u w}{\longrightarrow} \widetilde{\Lambda}\left(X^{*}\right), \\
A_{i \alpha}^{*} A_{j \alpha} \stackrel{u s}{\longrightarrow} A_{i}^{\prime \prime *} A_{j}^{\prime \prime}, \\
\Lambda\left(X_{\alpha}^{*} X_{\alpha}\right)=\sum_{i, j=1}^{n} \widetilde{\Lambda}\left(A_{i \alpha}^{*} A_{j \alpha} \otimes B_{i}^{*} B_{j}\right) \stackrel{u w}{\longrightarrow} \sum_{i, j=1}^{n} \widetilde{\Lambda}\left(A_{i}^{\prime \prime *} A_{j}^{\prime \prime} \otimes B_{i}^{*} B_{j}\right)=\widetilde{\Lambda}\left(X^{*} X\right),
\end{gathered}
$$

where $\stackrel{u w}{\longrightarrow}$ denotes the convergence in the ultraweak topology on $\mathscr{H}_{\mathscr{C}}$. Since $\Lambda$ is Schwarz, we obtain

$$
0 \leq\left(\begin{array}{cc}
\Lambda\left(X_{\alpha}^{*} X_{\alpha}\right) & \Lambda\left(X_{\alpha}^{*}\right) \\
\Lambda\left(X_{\alpha}\right) & \mathbb{1}_{\mathscr{C}}
\end{array}\right) \stackrel{u w}{\longrightarrow}\left(\begin{array}{cc}
\widetilde{\Lambda}\left(X^{*} X\right) & \widetilde{\Lambda}\left(X^{*}\right) \\
\widetilde{\Lambda}(X) & \mathbb{1}_{\mathscr{C}}
\end{array}\right)
$$

on $\mathscr{H}_{\mathscr{C}} \otimes \mathbb{C}^{2}$, which implies the Schwarz condition (4).

Lemma 5 Let $\mathscr{A}_{1}, \mathscr{A}_{2}, \mathscr{B}_{1}, \mathscr{B}_{2}$, and $\mathscr{C}$ be $C^{*}$-algebras, let $\Phi \in \mathbf{C h}^{\mathrm{CP}}\left(\mathscr{A}_{1} \rightarrow \mathscr{A}_{2}\right)$ and $\Psi \in \mathbf{C h} \mathbf{h P}^{\mathrm{CP}}\left(\mathscr{B}_{1} \rightarrow \mathscr{B}_{2}\right)$ be CP channels, and let $\Lambda \in \mathbf{C h}^{\mathrm{Sch}}\left(\mathscr{A}_{2} \otimes_{\mathrm{alg}} \mathscr{B}_{2} \rightarrow \mathscr{C}\right)$ be a Schwarz channel. Then $\Lambda \circ\left(\Phi \otimes_{\mathrm{alg}} \Psi\right) \in \mathbf{C h}^{\mathrm{Sch}}\left(\mathscr{A}_{1} \otimes_{\mathrm{alg}} \mathscr{B}_{1} \rightarrow \mathscr{C}\right)$.

Proof We first show that $\widetilde{\Lambda}:=\Lambda \circ\left(\Phi \otimes_{\text {alg }}\right.$ id $\left._{\mathscr{B}_{2}}\right): \mathscr{A}_{1} \otimes_{\text {alg }} \mathscr{B}_{2} \rightarrow \mathscr{C}$ is Schwarz. For this we have to show the Schwarz inequality

$$
\Lambda \circ \widetilde{\Phi}\left(X^{*} X\right) \geq \Lambda \circ \widetilde{\Phi}\left(X^{*}\right) \Lambda \circ \widetilde{\Phi}(X)
$$

for each $X \in \mathscr{A}_{1} \otimes_{\mathrm{alg}} \mathscr{B}_{2}$, where $\widetilde{\Phi}:=\Phi \otimes_{\text {alg }}$ id $_{\mathscr{B}_{2}}$. From the Schwarz condition of $\Lambda$, we have

$$
\Lambda\left(\widetilde{\Phi}\left(X^{*}\right) \widetilde{\Phi}(X)\right) \geq \Lambda \circ \widetilde{\Phi}\left(X^{*}\right) \Lambda \circ \widetilde{\Phi}(X) .
$$

Thus it is sufficient to show

$$
\Lambda\left(\widetilde{\Phi}\left(X^{*} X\right)-\widetilde{\Phi}\left(X^{*}\right) \widetilde{\Phi}(X)\right) \geq 0 .
$$

We write $X$ as $X=\sum_{i=1}^{n} A_{i} \otimes B_{i}\left(A_{i} \in \mathscr{A}_{1}, B_{i} \in \mathscr{B}_{2}\right)$. Since $\Phi$ is a CP channel, the map

$$
\Phi_{n}: \mathbb{M}_{n}\left(\mathscr{A}_{1}\right) \ni\left(A_{i j}\right)_{i, j=1}^{n} \mapsto\left(\Phi\left(A_{i j}\right)\right)_{i, j=1}^{n} \in \mathbb{M}_{n}\left(\mathscr{A}_{2}\right)
$$

is also a CP channel. Thus from the Schwarz inequality of $\Phi_{n}$, the matrix $\left(\Phi\left(A_{i}^{*} A_{j}\right)-\right.$ $\left.\Phi\left(A_{i}^{*}\right) \Phi\left(A_{j}\right)\right)_{i, j=1}^{n} \in \mathbb{M}_{n}\left(\mathscr{A}_{2}\right)$ is positive. Therefore there exists a matrix $\left(C_{i j}\right)_{i, j=1}^{n} \in$ $\mathbb{M}_{n}\left(\mathscr{A}_{2}\right)$ such that

$$
\Phi\left(A_{i}^{*} A_{j}\right)-\Phi\left(A_{i}^{*}\right) \Phi\left(A_{j}\right)=\sum_{k=1}^{n} C_{k i}^{*} C_{k j} .
$$


Thus we have

$$
\begin{aligned}
\widetilde{\Phi}\left(X^{*} X\right)-\widetilde{\Phi}\left(X^{*}\right) \widetilde{\Phi}(X) & =\sum_{i, j=1}^{n}\left(\Phi\left(A_{i}^{*} A_{j}\right)-\Phi\left(A_{i}^{*}\right) \Phi\left(A_{j}\right)\right) \otimes B_{i}^{*} B_{j} \\
& =\sum_{i, j, k=1}^{n} C_{k i}^{*} C_{k j} \otimes B_{i}^{*} B_{j} \\
& =\sum_{k=1}^{n} Y_{k}^{*} Y_{k}
\end{aligned}
$$

where $Y_{k}:=\sum_{i=1}^{n} C_{k i} \otimes B_{i}$. Consequently,

$$
(\text { LHS of (5) })=\sum_{k=1}^{n} \Lambda\left(Y_{k}^{*} Y_{k}\right) \geq \sum_{k=1}^{n} \Lambda\left(Y_{k}^{*}\right) \Lambda\left(Y_{k}\right) \geq 0
$$

Therefore $\widetilde{\Lambda}$ is Schwarz.

We can analogously show that $\Lambda \circ\left(\Phi \otimes_{\text {alg }} \Psi\right)=\widetilde{\Lambda} \circ\left(\operatorname{id}_{\mathscr{A}_{1}} \otimes_{\text {alg }} \Psi\right)$ is also Schwarz, which completes the proof.

Lemma 6 Let $\mathscr{E}=\left(\mathscr{M}, \Theta,\left(\varphi_{\theta}\right)_{\theta \in \Theta}\right), \mathscr{E}_{1}=\left(\mathscr{M}_{1}, \Theta,\left(\varphi_{\theta}^{(1)}\right)_{\theta \in \Theta}\right), \mathscr{F}=\left(\mathscr{N}, \Theta,\left(\psi_{\theta}\right)_{\theta \in \Theta}\right)$, and $\mathscr{F}_{1}=\left(\mathscr{N}_{1}, \Theta,\left(\psi_{\theta}^{(1)}\right)_{\theta \in \Theta}\right)$ be statistical experiments. Then we have the following.

1. $\mathscr{F} \Subset_{\text {min,bin }}^{\mathrm{CP}} \mathscr{E}$ and $\mathscr{F}_{1} \preccurlyeq \mathrm{CP}_{\sigma} \mathscr{F}$ imply $\mathscr{F}_{1} \Subset_{\text {min,bin }}^{\mathrm{CP}} \mathscr{E}$.

2. $\mathscr{F} \Subset_{\text {min,bin }}^{\mathrm{CP}} \mathscr{E}$ and $\mathscr{E} \sim \sim_{\mathrm{CP}_{\sigma}} \mathscr{E} 1$ imply $\mathscr{F} \Subset_{\text {min,bin }}^{\mathrm{CP}} \mathscr{E}$.

3. $\mathscr{F} \Subset_{\mathrm{alg}, \text { lnor }}^{\mathrm{Sch}} \mathscr{E}$ and $\mathscr{E} \sim \mathrm{CP}_{\sigma} \mathscr{E} 1$ imply $\mathscr{F} \Subset_{\mathrm{alg}, \text { lnor }}^{\mathrm{Sch}} \mathscr{E}$.

Proof 1. Let $\Lambda \in \mathbf{C h}^{\mathrm{CP}}\left(\mathscr{M} \otimes_{\min } \mathscr{N} \rightarrow \mathscr{M}\right)$ be a binormal channel that extracts $\mathscr{F}$ without disturbing $\mathscr{E}$ and let $\Gamma \in \mathbf{C h}_{\sigma}^{\mathrm{CP}}\left(\mathscr{N}_{1} \rightarrow \mathscr{N}\right)$ be a normal channel satisfying $\psi_{\theta}^{(1)}=\psi_{\theta} \circ \Gamma$ for all $\theta \in \Theta$. From Proposition 1 the tensor product map id $\otimes_{\min }$ $\Gamma \in \mathbf{C h}^{\mathrm{CP}}\left(\mathscr{M} \otimes_{\min } \mathscr{N}_{1} \rightarrow \mathscr{M} \otimes_{\min } \mathscr{N}\right)$ is well-defined. Then $\Lambda_{1}:=\Lambda \circ\left(\operatorname{id} \mathscr{M} \otimes_{\min }\right.$ $\Gamma) \in \mathbf{C h}^{\mathrm{CP}}\left(\mathscr{M} \otimes_{\min } \mathscr{N}_{1} \rightarrow \mathscr{M}\right)$ is a binormal channel that extracts $\mathscr{F}_{1}$ without disturbing $\mathscr{E}$, which proves $\mathscr{F}_{1} \Subset_{\text {min,bin }}^{\mathrm{CP}} \mathscr{E}$.

2. Let $\Lambda \in \mathbf{C h}^{\mathrm{CP}}\left(\mathscr{M} \otimes_{\min } \mathscr{N} \rightarrow \mathscr{M}\right)$ be a binormal channel that extracts $\mathscr{F}$ without disturbing $\mathscr{E}$ and let $\Phi \in \mathbf{C h}_{\sigma}^{\mathrm{CP}}\left(\mathscr{M}_{1} \rightarrow \mathscr{M}\right)$ and $\Psi \in \mathbf{C h}_{\sigma}^{\mathrm{CP}}\left(\mathscr{M} \rightarrow \mathscr{M}_{1}\right)$ be normal channels satisfying

$$
\begin{aligned}
& \varphi_{\theta}^{(1)}=\varphi_{\theta} \circ \Phi, \\
& \varphi_{\theta}=\varphi_{\theta}^{(1)} \circ \Psi
\end{aligned}
$$

for all $\theta \in \Theta$. From Proposition 11, the tensor product channel $\Phi \otimes_{\min } \mathrm{id}_{\mathscr{N}} \in$ $\mathbf{C h}^{\mathrm{CP}}\left(\mathscr{M}_{1} \otimes_{\min } \mathscr{N} \rightarrow \mathscr{M} \otimes_{\min } \mathscr{N}\right)$ is well-defined. We define the channel $\Lambda^{\prime}:=$ $\Psi \circ \Lambda \circ\left(\Phi \otimes_{\min } \mathrm{id}_{\mathscr{N}}\right) \in \mathbf{C h}^{\mathrm{CP}}\left(\mathscr{M}_{1} \otimes_{\min } \mathscr{N} \rightarrow \mathscr{M}_{1}\right)$. Then for each $\theta \in \Theta$, each 
$A \in \mathscr{M}_{1}$, and each $B \in \mathscr{N}$, we have

$$
\begin{aligned}
\Lambda^{\prime}(A \otimes B) & =\Psi \circ \Lambda(\Phi(A) \otimes B), \\
\varphi_{\theta}^{(1)} \circ \Lambda^{\prime}\left(A \otimes \mathbb{1}_{\mathscr{N}}\right) & =\varphi_{\theta}^{(1)} \circ \Psi \circ \Lambda\left(\Phi(A) \otimes \mathbb{1}_{\mathscr{N}}\right) \\
& =\varphi_{\theta} \circ \Lambda\left(\Phi(A) \otimes \mathbb{1}_{\mathscr{N}}\right) \\
& =\varphi_{\theta} \circ \Phi(A) \\
& =\varphi_{\theta}^{(1)}(A), \\
\varphi_{\theta}^{(1)} \circ \Lambda^{\prime}\left(\mathbb{1}_{\mathscr{M}_{1}} \otimes B\right) & =\varphi_{\theta}^{(1)} \Psi \circ \Lambda\left(\Phi\left(\mathbb{1}_{\mathscr{M}_{1}}\right) \otimes B\right) \\
& =\varphi_{\theta} \circ \Lambda\left(\mathbb{1}_{\mathscr{M}} \otimes B\right) \\
& =\psi_{\theta}(B) .
\end{aligned}
$$

Therefore $\Lambda^{\prime}$ is a binormal CP channel that extracts $\mathscr{F}$ without disturbing $\mathscr{E} 1$, which proves $\mathscr{F} \Subset_{\text {min,bin }}^{\mathrm{CP}} \mathscr{E}$.

3. Let $\Lambda \in \mathbf{C h}^{\mathrm{Sch}}\left(\mathscr{M} \otimes_{\text {alg }} \mathscr{N} \rightarrow \mathscr{M}\right)$ be a left-normal Schwarz channel that extracts $\mathscr{F}$ without disturbing $\mathscr{E}$ and let $\Phi \in \mathbf{C h}_{\sigma}^{\mathrm{CP}}\left(\mathscr{M}_{1} \rightarrow \mathscr{M}\right)$ and $\Psi \in \mathbf{C h}_{\sigma}^{\mathrm{CP}}\left(\mathscr{M} \rightarrow \mathscr{M}_{1}\right)$ be normal channels satisfying (6) and (7). From Lemma 5 we have $\Lambda \circ\left(\Phi \otimes_{\text {alg }}\right.$ $\left.\operatorname{id}_{\mathscr{N}}\right) \in \mathbf{C h}^{\text {Sch }}\left(\mathscr{M}_{1} \otimes_{\text {alg }} \mathscr{N} \rightarrow \mathscr{M}\right)$, and hence $\Lambda^{\prime}:=\Psi \circ \Lambda \circ\left(\Phi \otimes_{\text {alg }} \operatorname{id}_{\mathscr{N}}\right) \in$ $\mathbf{C h}^{\mathrm{Sch}}\left(\mathscr{M}_{1} \otimes_{\text {alg }} \mathscr{N} \rightarrow \mathscr{M}_{1}\right)$. Then we can show that $\Lambda^{\prime}$ is a left-normal Schwarz channel that extracts $\mathscr{F}$ without disturbing $\mathscr{E}_{1}$ in the same way as in the proof of claim 2. Thus $\mathscr{F} \Subset_{\text {alg, } \operatorname{lnor}}^{\mathrm{Sch}} \mathscr{E} 1$.

Lemma 7 Let $\mathscr{E}=\left(\mathscr{M}, \Theta,\left(\varphi_{\theta}\right)_{\theta \in \Theta}\right)$ be a statistical experiment. Define

$$
\mathscr{F}:=\left(\mathfrak{Z}(\mathscr{M}), \Theta,\left(\psi_{\theta}\right)_{\theta \in \Theta}\right),
$$

where $\psi_{\theta}$ is the restriction of $\varphi_{\theta}$ to the center $\mathfrak{Z}(\mathscr{M})$. Then $\mathscr{F} \Subset_{\text {min,bin }}^{\mathrm{CP}} \mathscr{E}$.

Proof Since $\mathscr{M}$ and $\mathfrak{Z}(\mathscr{M})$ commute, there exists a representation $\pi: \mathscr{M} \otimes_{\max } \mathfrak{Z}(\mathscr{M})=$ $\mathscr{M} \otimes \min \mathfrak{Z}(\mathscr{M}) \rightarrow \mathscr{M}$ such that $\pi(A \otimes Z)=A Z(A \in \mathscr{M}, Z \in \mathfrak{Z}(\mathscr{M}))$. Then for each $A \in \mathscr{M}$, each $Z \in \mathfrak{Z}(\mathscr{M})$, and each $\theta \in \Theta$, we have

$$
\begin{gathered}
\varphi_{\theta} \circ \pi(A \otimes \mathbb{1} \mathscr{M})=\varphi_{\theta}(A), \\
\varphi_{\theta} \circ \pi\left(\mathbb{1}_{\mathscr{M}} \otimes Z\right)=\varphi_{\theta}(Z)=\psi_{\theta}(Z) .
\end{gathered}
$$

Therefore $\pi \in \mathbf{C h}^{\mathrm{CP}}\left(\mathscr{M} \otimes_{\min } \mathfrak{Z}(\mathscr{M}) \rightarrow \mathscr{M}\right)$ is a binormal channel that extracts $\mathscr{F}$ without disturbing $\mathscr{E}$, which proves $\mathscr{F} \Subset_{\text {min,bin }}^{\mathrm{CP}} \mathscr{E}$.

Proof of Theorem $\square$ Let $\mathscr{M} \otimes \gamma \mathscr{N}$ be an arbitrary $C^{*}$-tensor product of $\mathscr{M}$ and $\mathscr{N}$, let $\mathscr{E}_{0}=\left(\mathscr{M}_{0}, \Theta,\left(\varphi_{\theta}^{(0)}\right)_{\theta \in \Theta}\right)$ be the minimal sufficient statistical experiment satisfying $\mathscr{E} 0 \sim \mathrm{CP}_{\sigma} \mathscr{E}$, and let $\mathscr{E}_{\mathrm{cl}}=\left(\mathfrak{Z}\left(\mathscr{M}_{0}\right), \Theta,\left(\varphi_{\theta}^{\mathrm{cl}}\right)_{\theta \in \Theta}\right)$ be the classical part of $\mathscr{E}$. From the definitions of the accessibility relations $\Subset_{y, z}^{X}$, the following implications immediately follow:

$$
\begin{gathered}
\mathscr{F} \Subset_{y, z}^{\mathrm{CP}} \mathscr{E} \Longrightarrow \mathscr{F} \Subset_{y, z}^{\mathrm{Sch}} \mathscr{E}, \\
\mathscr{F} \Subset_{\gamma, z}^{X} \mathscr{E} \Longrightarrow \mathscr{F} \Subset_{\mathrm{alg}, z}^{X} \mathscr{E}, \\
\mathscr{F} \Subset_{y, \text { bin }}^{X} \mathscr{E} \Longrightarrow \mathscr{F} \Subset_{y, \text { lnor }}^{X} \mathscr{E} \Longrightarrow \mathscr{F} \Subset_{y}^{X} \mathscr{E} .
\end{gathered}
$$


Hence

$$
\mathscr{F} \Subset_{\gamma, \text { bin }}^{\mathrm{CP}} \mathscr{E} \Longrightarrow \mathscr{F} \Subset_{y, z}^{X} \mathscr{E} \Longrightarrow \mathscr{F} \Subset_{\text {alg }}^{\mathrm{Sch}} \mathscr{E}
$$

if $y=\gamma$ or $y=$ alg. Thus it is sufficient to establish the implications (i) $\Longrightarrow$ (ii) $\Longrightarrow$ (iii) $\Longrightarrow$ (iv) $\Longrightarrow(\mathrm{v})$ for the following conditions:

(i) $\mathscr{F} \Subset_{\mathrm{alg}}^{\mathrm{Sch}} \mathscr{E}$;

(ii) $\mathscr{F} \Subset_{\text {alg.lnor }}^{\mathrm{Sch}} \mathscr{E}$;

(iii) $\mathscr{F} \preccurlyeq \mathrm{CP}_{\sigma} \mathscr{E}_{\mathrm{cl}}$;

(iv) $\mathscr{F} \Subset_{\text {min,bin }}^{\mathrm{CP}} \mathscr{E}$;

(v) $\mathscr{F} \Subset_{\gamma, \text { bin }}^{\mathrm{CP}} \mathscr{E}$.

(i) $\Longrightarrow$ (ii). Assume (i) and take a channel $\Lambda \in \mathbf{C h}^{\mathrm{Sch}}\left(\mathscr{M} \otimes_{\text {alg }} \mathscr{N} \rightarrow \mathscr{M}\right)$ that extracts $\mathscr{F}$ without disturbing $\mathscr{E}$. Then from Lemma $4, \Lambda$ extends to a left-normal channel $\widetilde{\Lambda} \in \mathbf{C h}^{\mathrm{Sch}}\left(\mathscr{M}^{* *} \otimes_{\text {alg }} \mathscr{N} \rightarrow \mathscr{M}^{* *}\right)$. If we write the normal extension of $\mathscr{E}$ as $\overline{\mathscr{E}}=\left(\mathscr{M}^{* *}, \Theta,\left(\overline{\varphi_{\theta}}\right)_{\theta \in \Theta}\right)$, then for each $A \in \mathscr{M}$, each $B \in \mathscr{N}$, and each $\theta \in \Theta$, we have

$$
\begin{gathered}
\overline{\varphi_{\theta}} \circ \widetilde{\Lambda}\left(A \otimes \mathbb{1}_{\mathscr{N}}\right)=\varphi_{\theta} \circ \Lambda\left(A \otimes \mathbb{1}_{\mathscr{N}}\right)=\varphi_{\theta}(A)=\overline{\varphi_{\theta}}(A), \\
\overline{\varphi_{\theta}} \circ \widetilde{\Lambda}\left(\mathbb{1}_{\mathscr{M}^{* *}} \otimes B\right)=\varphi_{\theta} \circ \Lambda\left(\mathbb{1}_{\mathscr{M}} \otimes B\right)=\psi_{\theta}(B) .
\end{gathered}
$$

Since $\mathscr{M}$ is ultraweakly dense in $\mathscr{M}^{* *}$ and $\widetilde{\Lambda}$ is left-normal, (8) implies

$$
\overline{\varphi_{\theta}} \circ \widetilde{\Lambda}\left(A^{\prime \prime} \otimes \mathbb{1}_{\mathscr{N}}\right)=\overline{\varphi_{\theta}}\left(A^{\prime \prime}\right)
$$

for all $A^{\prime \prime} \in \mathscr{M}^{* *}$. Therefore we obtain $\mathscr{F} \Subset_{\text {alg,lnor }}^{\text {Sch }} \overline{\mathscr{E}}$. From Lemmas 2 and 6 , this implies $\mathscr{F} \Subset_{\text {alg,lnor }}^{\text {Sch }} \mathscr{E}$.

(ii) $\Longrightarrow$ (iii). Assume (ii). From Lemma 6 we have $\mathscr{F} \Subset_{\text {alg.lnor }}^{\text {Sch }} \mathscr{E} 0$ and hence we can take a left-normal channel $\Lambda \in \mathbf{C h}^{\text {Sch }}\left(\mathscr{M}_{0} \otimes_{\text {alg }} \mathscr{N} \rightarrow \mathscr{M}_{0}\right)$ that extracts $\mathscr{F}$ without disturbing $\mathscr{E}_{0}$. We define Schwarz channels $\Lambda_{L} \in \mathbf{C h}_{\sigma}^{\mathrm{Sch}}\left(\mathscr{M}_{0}\right)$ and $\Lambda_{R} \in \mathbf{C h}^{\mathrm{Sch}}(\mathscr{N} \rightarrow$ $\left.\mathscr{M}_{0}\right)$ by $\Lambda_{L}(A):=\Lambda\left(A \otimes \mathbb{1}_{\mathscr{N}}\right)$ and $\Lambda_{R}(B):=\Lambda\left(\mathbb{1}_{\mathscr{M}_{0}} \otimes B\right)\left(A \in \mathscr{M}_{0}, B \in \mathscr{N}\right)$. Since we have $\varphi_{\theta}^{(0)} \circ \Lambda_{L}=\varphi_{\theta}^{(0)}$ for all $\theta \in \Theta$, the minimal sufficiency of $\mathscr{E}_{0}$ implies $\Lambda_{L}=$ $\operatorname{id}_{\mathscr{M}_{0}}$, i.e. $\Lambda\left(A \otimes \mathbb{1}_{\mathscr{N}}\right)=A$ for all $A \in \mathscr{M}_{0}$. Thus for each $A \in \mathscr{M}_{0}$ we have

$$
\begin{aligned}
& \Lambda\left(\left(A \otimes \mathbb{1}_{\mathscr{N}}\right)^{*}\left(A \otimes \mathbb{1}_{\mathscr{N}}\right)\right)=A^{*} A=\Lambda\left(A \otimes \mathbb{1}_{\mathscr{N}}\right)^{*} \Lambda\left(A \otimes \mathbb{1}_{\mathscr{N}}\right), \\
& \Lambda\left(\left(A \otimes \mathbb{1}_{\mathscr{N}}\right)\left(A \otimes \mathbb{1}_{\mathscr{N}}\right)^{*}\right)=A A^{*}=\Lambda\left(A \otimes \mathbb{1}_{\mathscr{N}}\right) \Lambda\left(A \otimes \mathbb{1}_{\mathscr{N}}\right)^{*} .
\end{aligned}
$$

Therefore $\mathscr{M}_{0} \otimes \mathbb{1}_{\mathscr{N}}$ is contained in the multiplicative domain of $\Lambda$. Thus, from Lemma1, for each $A \in \mathscr{M}_{0}$ and each $B \in \mathscr{N}$, we have

$$
\begin{aligned}
A \Lambda_{R}(B) & =\Lambda\left(A \otimes \mathbb{1}_{\mathscr{N}}\right) \Lambda\left(\mathbb{1}_{\mathscr{M}_{0}} \otimes B\right) \\
& =\Lambda(A \otimes B) \\
& =\Lambda\left(\mathbb{1}_{\mathscr{M}_{0}} \otimes B\right) \Lambda\left(A \otimes \mathbb{1}_{\mathscr{N}}\right) \\
& =\Lambda_{R}(B) A
\end{aligned}
$$


which implies $\Lambda_{R}(\mathscr{N}) \subseteq \mathfrak{Z}\left(\mathscr{M}_{0}\right)$. Since $\mathfrak{Z}\left(\mathscr{M}_{0}\right)$ is commutative, $\Lambda_{R}$ is CP. Furthermore, we have $\psi_{\theta}=\varphi_{\theta}^{(0)} \circ \Lambda_{R}=\varphi_{\theta}^{\mathrm{cl}} \circ \Lambda_{R}$ for all $\theta \in \Theta$, which implies $\mathscr{F} \preccurlyeq \mathrm{CP} \mathscr{E}_{\mathrm{cl}}$. Thus from Lemma 3 we obtain $\mathscr{F} \preccurlyeq \mathrm{CP}_{\sigma} \mathscr{E}_{\mathrm{cl}}$.

(iii) $\Longrightarrow$ (iv) follows from Lemmas 6 and 7

(iv) $\Longrightarrow$ (v). Assume (iv) and let $\Lambda \in \mathbf{C h}^{\mathrm{CP}}\left(\mathscr{M} \otimes_{\min } \mathscr{N} \rightarrow \mathscr{M}\right)$ be a binormal CP channel that extracts $\mathscr{F}$ without disturbing $\mathscr{E}$. From the minimality of the norm $\|\cdot\|_{\text {min }}$ on $\mathscr{M} \otimes_{\text {alg }} \mathscr{N}$, there exists a representation $\pi_{\min }: \mathscr{M} \otimes_{\gamma} \mathscr{N} \rightarrow \mathscr{M} \otimes_{\min } \mathscr{N}$ such that $\pi_{\min }(A \otimes B)=A \otimes B(A \in \mathscr{M}, B \in \mathscr{N})$. We define a channel $\Lambda_{\gamma} \in \mathbf{C h}^{\mathrm{CP}}\left(\mathscr{M} \otimes_{\gamma}\right.$ $\mathscr{N} \rightarrow \mathscr{M})$ by $\Lambda_{\gamma}:=\Lambda \circ \pi_{\min }$. Then $\Lambda_{\gamma}$ is a binormal channel that extracts $\mathscr{F}$ without disturbing $\mathscr{E}$, which implies $\mathscr{F} \Subset_{\gamma, \text { bin }}^{\mathrm{CP}} \mathscr{E}$.

Remark 1 The proof of (ii) $\Longrightarrow$ (iii) in Theorem 1 is analogous to the proof of Lindblad's "general no-cloning theorem" ([16], Theorem 1), in which the set of invariant states of a given broadcasting channel is considered.

Since all the relations $\mathscr{F} \Subset_{y, Z}^{X} \mathscr{E}$ in Definition 1 coincide, from now on we adopt the simpler notation $\mathscr{F} \Subset \mathscr{E}$ instead of $\mathscr{F} \Subset_{y, Z}^{X} \mathscr{E}$.

\subsection{No-broadcasting}

A statistical experiment $\mathscr{E}=\left(\mathscr{M}, \Theta,\left(\varphi_{\theta}\right)_{\theta \in \Theta}\right)$ is called broadcastable in the sense of algebraic tensor product if there exists a Schwarz channel $\Lambda \in \mathbf{C h}^{\mathrm{Sch}}\left(\mathscr{M} \otimes_{\text {alg }} \mathscr{M} \rightarrow\right.$ $\mathscr{M})$ such that

$$
\varphi_{\theta}(A)=\varphi_{\theta} \circ \Lambda\left(A \otimes \mathbb{1}_{\mathscr{M}}\right)=\varphi_{\theta} \circ \Lambda\left(\mathbb{1}_{\mathscr{M}} \otimes A\right)
$$

for all $\theta \in \Theta$ and all $A \in \mathscr{M}$. From the definition, we can easily see that $\mathscr{E}$ is broadcastable in the sense of algebraic tensor product if and only if $\mathscr{E} \Subset \mathscr{E}$. Therefore from Theorem 1 we immediately obtain

Corollary 1 (No-broadcasting theorem) A statistical experiment $\mathscr{E}$ is broadcastable in the sense of algebraic tensor product if and only if $\mathscr{E}$ is normally CP equivalent to a classical statistical experiment.

Remark 2 In [10], the broadcastability of normal states was considered by identifying the outcome composite system with the normal tensor product $\mathscr{M} \bar{\otimes} \mathscr{M}$, which is more restrictive than our broadcastability condition here. Indeed, a statistical experiment $\mathscr{E}$ is broadcastable in the sense of [10] if and only if $\mathscr{E}$ is normally CP equivalent to a classical statistical experiment with an atomic outcome von Neumann algebra, and hence any minimal sufficient statistical experiment with a non-atomic commutative outcome algebra is not broadcastable in this sense. For the difference of the $C^{*}$ - and normal tensor products from the view point of broadcasting, see also Section 7 of [15]. 


\subsection{Density operators}

Let us consider a statistical experiment $\mathscr{E}=\left(\mathscr{L}(\mathscr{H}), \Theta,\left(\varphi_{\theta}\right)_{\theta \in \Theta}\right)$ for a Hilbert space $\mathscr{H}$. As usual we regard each normal state $\varphi_{\theta}$ as a density operator $\rho_{\theta}$ on $\mathscr{H}$ satisfying $\varphi_{\theta}(A)=\operatorname{tr}\left(\rho_{\theta} A\right)(A \in \mathscr{L}(\mathscr{H}))$. By restricting the outcome Hilbert space $\mathscr{H}$ if necessary, we assume that $\mathscr{E}$ is faithful. Let $\mathscr{M}_{0} \subseteq \mathscr{L}(\mathscr{H})$ be the minimal sufficient subalgebra with respect to $\left(\varphi_{\theta}\right)_{\theta \in \Theta}$ and let $\mathbb{E}$ be the normal conditional expectation from $\mathscr{L}(\mathscr{H})$ onto $\mathscr{M}_{0}$ satisfying $\varphi_{\theta} \circ \mathbb{E}=\varphi_{\theta}$ for all $\theta \in \Theta$. Since $\left(\varphi_{\theta}\right)_{\theta \in \Theta}$ is faithful, so is $\mathbb{E}$. Hence, according to [20] (Chapter V, Section 2, Exercise 8) $\mathscr{M}_{0}$ is atomic, i.e., we have the following decompositions:

$$
\begin{gathered}
\mathscr{H}=\bigoplus_{i \in I} \mathscr{H}_{i} \otimes \mathscr{K}_{i}, \\
\mathscr{M}_{0}=\bigoplus_{i \in I} \mathscr{L}\left(\mathscr{H}_{i}\right) \bar{\otimes} \mathbb{C} \mathbb{K}_{\mathscr{K}_{i}},
\end{gathered}
$$

where $\mathscr{H}_{i}$ and $\mathscr{K}_{i}$ are Hilbert spaces and $\mathbb{1}_{\mathscr{K}}$ denotes the identity operator on a Hilbert space $\mathscr{K}$. (We can also show this from [19]). Then, as in the finite-dimensional case ([16], Section 4; [7], Appendix A), we can show the following decompositions for $\mathbb{E}$ and $\rho_{\theta}$ :

$$
\begin{gathered}
\mathbb{E}(A)=\bigoplus_{i \in I} \operatorname{tr}_{\mathscr{K}_{i}}\left(P_{i} A P_{i}\left(\mathbb{1}_{\mathscr{H}_{i}} \otimes \sigma_{i}\right)\right) \otimes \mathbb{1}_{\mathscr{K}_{i}} \quad(A \in \mathscr{L}(\mathscr{H})), \\
\rho_{\theta}=\bigoplus_{i \in I} q_{\theta}(i) \rho_{i, \theta} \otimes \sigma_{i},
\end{gathered}
$$

where $P_{i}$ is the orthogonal projection onto $\mathscr{H}_{i} \otimes \mathscr{K}_{i}, \operatorname{tr}_{\mathscr{K}_{i}}(\cdot)$ is the partial trace over $\mathscr{K}_{i}$, $q_{\theta}(i)$ is a probability distribution over $i \in I$ for each $\theta \in \Theta$, and $\rho_{i, \theta}$ and $\sigma_{i}$ are density operators on $\mathscr{H}_{i}$ and $\mathscr{K}_{i}$, respectively. If $\mathscr{H}$ is finite-dimensional, the decomposition given by (9) and (10) coincides with the maximal decomposition obtained by Koashi and Imoto [12].

Since we have $\mathfrak{Z}\left(\mathscr{M}_{0}\right)=\bigoplus_{i \in I} \mathbb{C} P_{i}$, the classical part $\mathscr{E}_{\mathrm{cl}}$ is normally isomorphic to $\left(\ell^{\infty}(I), \Theta,\left(Q_{\theta}\right)_{\theta \in \Theta}\right)$, where $\ell^{\infty}(I)$ is the set of bounded complex-valued functions on $I$ and $Q_{\theta} \in \mathscr{S}_{\sigma}\left(\ell^{\infty}(I)\right)$ is given by $Q_{\theta}(f)=\sum_{i \in I} q_{\theta}(i) f(i)\left(f \in \ell^{\infty}(I)\right)$. In this sense, the classical part $\mathscr{E}_{\mathrm{cl}}$ corresponds to the probability distributions $q_{\theta}(i)$ appearing in the maximal decomposition (10). We can extract, in this case, the information $\mathscr{E}_{\mathrm{cl}}$ without disturbing $\mathscr{E}$ by performing the discrete projective measurement corresponding to $\left(P_{i}\right)_{i \in I}$.

We remark that the infinite-dimensional version of the Koashi-Imoto decomposition was obtained in [9] for separable $\mathscr{H}$ by using the modular theory in operator algebras.

\section{Direct product of statistical experiments}

In this section we consider the direct product of statistical experiments and its classical part. 
Let $\mathscr{E}=\left(\mathscr{M}, \Theta,\left(\varphi_{\theta}\right)_{\theta \in \Theta}\right)$ and $\mathscr{F}=\left(\mathscr{N}, \Xi,\left(\psi_{\xi}\right)_{\xi \in \Xi}\right)$ be statistical experiments. We define the direct product $\mathscr{E} \otimes \mathscr{F}$ by

$$
\mathscr{E} \otimes \mathscr{F}:=\left(\mathscr{M} \bar{\otimes} \mathscr{N}, \Theta \times \Xi,\left(\varphi_{\theta} \bar{\otimes} \psi_{\xi}\right)_{(\theta, \xi) \in \Theta \times \Xi}\right) .
$$

Operationally, $\mathscr{E} \otimes \mathscr{F}$ corresponds to the juxtaposition of two partially known systems corresponding to $\mathscr{E}$ and $\mathscr{F}$.

We first show that the direct product of minimal sufficient statistical experiments is also minimal sufficient.

Theorem 2 Let $\mathscr{E}=\left(\mathscr{M}, \Theta,\left(\varphi_{\theta}\right)_{\theta \in \Theta}\right)$ and $\mathscr{F}=\left(\mathscr{N}, \Xi,\left(\psi_{\xi}\right)_{\xi \in \Xi}\right)$ be statistical experiments. Then the following conditions are equivalent:

(i) $\mathscr{E} \otimes \mathscr{F}$ is minimal sufficient;

(ii) $\mathscr{E}$ and $\mathscr{F}$ are minimal sufficient.

Proof (i) $\Longrightarrow$ (ii). Assume (i). Let $\Phi \in \mathbf{C h}_{\sigma}^{\mathrm{CP}}(\mathscr{M})$ and $\Psi \in \mathbf{C h}_{\sigma}^{\mathrm{CP}}(\mathscr{N})$ be normal channels satisfying $\varphi_{\theta} \circ \Phi=\varphi_{\theta}(\theta \in \Theta)$ and $\psi_{\xi} \circ \Psi=\psi_{\xi}(\xi \in \Xi)$. Then we have

$$
\left(\varphi_{\theta} \bar{\otimes} \psi_{\xi}\right) \circ(\Phi \bar{\otimes} \Psi)=\left(\varphi_{\theta} \circ \Phi\right) \bar{\otimes}\left(\psi_{\xi} \circ \Psi\right)=\varphi_{\theta} \bar{\otimes} \psi_{\xi}
$$

for each $(\theta, \xi) \in \Theta \times \Xi$. Thus from the minimal sufficiency of $\mathscr{E} \otimes \mathscr{F}$, we have $\Phi \bar{\otimes} \Psi=\mathrm{id}_{\mathscr{M} \Phi \mathscr{N}}$, which implies $\Phi=\mathrm{id}_{\mathscr{M}}$ and $\Psi=\mathrm{id}_{\mathscr{N}}$. Therefore $\mathscr{E}$ and $\mathscr{F}$ are minimal sufficient.

(ii) $\Longrightarrow$ (i). Assume (ii). Since we have $\mathrm{s}\left(\varphi_{\theta} \bar{\otimes} \psi_{\xi}\right)=\mathrm{s}\left(\varphi_{\theta}\right) \otimes \mathrm{s}\left(\psi_{\xi}\right)$ ([20], Corollary IV.5.12), the family $\left(\varphi_{\theta} \bar{\otimes} \psi_{\xi}\right)_{(\theta, \xi) \in \Theta \times \Xi}$ is faithful on $\mathscr{M} \bar{\otimes} \mathscr{N}$. Therefore, by applying the mean ergodic theorem for von Neumann algebras, there exists a minimal sufficient subalgebra $\mathscr{M}_{0} \subseteq \mathscr{M} \bar{\otimes} \mathscr{N}$ with respect to $\left(\varphi_{\theta} \bar{\otimes} \psi_{\xi}\right)_{(\theta, \xi) \in \Theta \times \Xi}$ and a normal conditional expectation $\mathbb{E}$ from $\mathscr{M} \bar{\otimes} \mathscr{N}$ onto $\mathscr{M}_{0}$ such that $\left(\varphi_{\theta} \bar{\otimes} \psi_{\xi}\right) \circ \mathbb{E}=\varphi_{\theta} \bar{\otimes} \psi_{\xi}$ $(\theta \in \Theta, \xi \in \Xi)$. We will show $\mathscr{M} \bar{\otimes} \mathscr{N} \subseteq \mathscr{M}_{0}$, which implies the condition (i).

First, we fix arbitrary $\xi \in \Xi$ and write $\mathrm{s}_{\xi}:=\mathrm{s}\left(\psi_{\xi}\right)$ and $\tilde{s}_{\xi}:=\mathbb{1}_{\mathscr{M}} \otimes \mathrm{s}\left(\psi_{\xi}\right)$. Consider the following faithful statistical experiment:

$$
\widetilde{\mathscr{E}}_{\xi}:=\left(\mathscr{M} \bar{\otimes}\left(\mathrm{s}_{\xi} \mathscr{N} \mathrm{s}_{\xi}\right), \Theta,\left(\varphi_{\theta} \bar{\otimes} \psi_{\xi}\right)_{\theta \in \Theta}\right) .
$$

Define a map $\mathbb{E}_{\xi}: \mathscr{M} \bar{\otimes}\left(\mathrm{s}_{\xi} \mathscr{N} \mathrm{s}_{\xi}\right) \rightarrow \mathscr{M} \bar{\otimes} \mathbb{C s}_{\xi}$ by the normal extension of $\mathbb{E}_{\xi}(A \otimes$ $B)=A \otimes \psi_{\xi}(B) \mathrm{s}_{\xi}\left(A \in \mathscr{M}, B \in \mathrm{s}_{\xi} \mathscr{N} \mathrm{s}_{\xi}\right)$. Then $\mathbb{E}_{\xi}$ is a normal conditional expectation from $\mathscr{M} \bar{\otimes}\left(\mathrm{s}_{\xi} \mathscr{N} \mathrm{s}_{\xi}\right)$ onto $\mathscr{M} \bar{\otimes} \mathbb{C} s_{\xi}$ and satisfies

$$
\left(\varphi_{\theta} \bar{\otimes} \psi_{\xi}\right) \circ \mathbb{E}_{\xi}=\varphi_{\theta} \bar{\otimes} \psi_{\xi}
$$

for all $\theta \in \Theta$. Since the statistical experiment

$$
\left(\mathscr{M} \bar{\otimes} \mathbb{C s}_{\xi}, \Theta,\left(\varphi_{\theta} \bar{\otimes} \psi_{\xi}\right)_{\theta \in \Theta}\right)
$$

is normally isomorphic to $\mathscr{E}$, the assumption of the minimal sufficiency of $\mathscr{E}$ implies that $\mathscr{M} \bar{\otimes} \mathrm{Cs}_{\xi}$ is the minimal sufficient subalgebra of $\mathscr{M} \bar{\otimes}\left(\mathrm{s}_{\xi} \mathscr{N} \mathrm{s}_{\xi}\right)$ with respect to the family $\left(\varphi_{\theta} \bar{\otimes} \psi_{\xi}\right)_{\theta \in \Theta}([14]$, Theorem 3). Therefore for a normal channel $\Gamma \in$ $\mathbf{C h}_{\sigma}^{\mathrm{CP}}\left(\mathscr{M} \bar{\otimes}\left(\mathrm{s}_{\xi} \mathscr{N} \mathrm{s}_{\xi}\right)\right),\left(\varphi_{\theta} \bar{\otimes} \psi_{\xi}\right) \circ \Gamma(X)=\left(\varphi_{\theta} \bar{\otimes} \psi_{\xi}\right)(X)\left(X \in \mathscr{M} \bar{\otimes}\left(\mathrm{s}_{\xi} \mathscr{N} \mathrm{s}_{\xi}\right), \theta \in\right.$ $\Theta)$ implies $\Gamma\left(A \otimes \mathrm{s}_{\xi}\right)=A \otimes \mathrm{s}_{\xi}(A \in \mathscr{M})$. 
Since $\mathrm{s}\left(\varphi_{\theta}\right) \otimes \mathrm{s}\left(\psi_{\xi}\right)=\mathrm{s}\left(\varphi_{\theta} \bar{\otimes} \psi_{\xi}\right) \in \mathscr{M}_{0}$ for each $(\theta, \xi) \in \Theta \times \Xi([21]$, Lemma 1$)$, we have $\tilde{\mathrm{s}}_{\xi}=\bigvee_{\theta \in \Theta} \mathrm{s}\left(\varphi_{\theta}\right) \otimes \mathrm{s}\left(\psi_{\xi}\right) \in \mathscr{M}_{0}$. Therefore we obtain

$$
\begin{gathered}
\mathbb{E}\left(\tilde{\mathrm{s}}_{\xi}\right)=\tilde{\mathrm{s}}_{\xi}, \\
\mathbb{E}(X)=\mathbb{E}\left(\tilde{\mathrm{s}}_{\xi} X \tilde{\mathrm{s}}_{\xi}\right)=\tilde{\mathrm{s}}_{\xi} \mathbb{E}(X) \tilde{\mathrm{s}}_{\xi} \in \mathscr{M} \otimes\left(\mathrm{s}_{\xi} \mathscr{N} \mathrm{s}_{\xi}\right) \quad\left(X \in \mathscr{M} \bar{\otimes}\left(\mathrm{s}_{\xi} \mathscr{N} \mathrm{s}_{\xi}\right)\right) .
\end{gathered}
$$

Thus if we define $\Lambda_{\xi}$ by the restriction of $\mathbb{E}$ to $\mathscr{M} \bar{\otimes}(\mathrm{s} \xi \mathscr{N} \mathrm{s} \xi), \Lambda_{\xi}$ is a normal channel on $\mathscr{M} \bar{\otimes}\left(\mathrm{s}_{\xi} \mathscr{N} \mathrm{s}_{\xi}\right)$. Then $\left(\varphi_{\theta} \bar{\otimes} \psi_{\xi}\right) \circ \Lambda_{\xi}(X)=\left(\varphi_{\theta} \bar{\otimes} \psi_{\xi}\right) \circ \mathbb{E}(X)=\left(\varphi_{\theta} \bar{\otimes} \psi_{\xi}\right)(X)$ for each $\theta \in \Theta$ and each $X \in \mathscr{M} \bar{\otimes}\left(\mathrm{s}_{\xi} \mathscr{N} \mathrm{s} \xi\right)$. Therefore for each $A \in \mathscr{M}$ we have

$$
\mathscr{M}_{0} \ni \mathbb{E}(A \otimes \mathrm{s} \xi)=\Lambda_{\xi}(A \otimes \mathrm{s} \xi)=A \otimes \mathrm{s} \xi .
$$

Hence we obtain $\mathscr{M} \bar{\otimes} \mathbb{C s}_{\xi} \subseteq \mathscr{M}_{0}$ for each $\xi \in \Xi$. Thus for each projection $P \in \mathscr{M}$ we have

$$
P \otimes \mathbb{1}_{\mathscr{N}}=\bigvee_{\xi \in \Xi} P \otimes \mathrm{s}_{\xi} \in \mathscr{M}_{0},
$$

which implies $\mathscr{M} \otimes \mathbb{1}_{\mathscr{N}} \subseteq \mathscr{M}_{0}$. We can also show $\mathbb{1}_{\mathscr{M}} \otimes \mathscr{N} \subseteq \mathscr{M}_{0}$ analogously. Thus we finally obtain $\mathscr{M} \bar{\otimes} \mathscr{N} \subseteq \mathscr{M}_{0}$, which completes the proof.

Remark 3 A corresponding result of Theorem 2 for dominated families of probability measures was obtained in [4] and for families of finite-dimensional density operators in [12] (Theorem 4).

By using Theorem 2, we show that the classical part of a direct product of statistical experiments is the direct product of the classical parts of the statistical experiments:

Theorem 3 Let $\mathscr{E}=\left(\mathscr{M}, \Theta,\left(\varphi_{\theta}\right)_{\theta \in \Theta}\right)$ and $\mathscr{F}=\left(\mathscr{N}, \Xi,\left(\psi_{\xi}\right)_{\xi \in \Xi}\right)$ be statistical experiments. Then $(\mathscr{E} \otimes \mathscr{F})_{\mathrm{cl}} \cong \mathscr{E}_{\mathrm{cl}} \otimes \mathscr{F}_{\mathrm{cl}}$.

Proof Let $\mathscr{E}_{0}=\left(\mathscr{M}_{0}, \Theta,\left(\varphi_{\theta}^{(0)}\right)_{\theta \in \Theta}\right)$ and $\mathscr{F}_{0}=\left(\mathscr{N}_{0}, \Xi,\left(\psi_{\xi}^{(0)}\right)_{\xi \in \Xi}\right)$ be the minimal sufficient statistical experiments satisfying $\mathscr{E} \sim \mathrm{CP}_{\sigma} \mathscr{E} 0$ and $\mathscr{F} \sim \mathrm{CP}_{\sigma} \mathscr{F} 0$, and let $\mathscr{E}_{\mathrm{cl}}=$ $\left(\mathfrak{Z}\left(\mathscr{M}_{0}\right), \Theta,\left(\varphi_{\theta}^{\mathrm{cl}}\right)_{\theta \in \Theta}\right)$ and $\mathscr{F}_{\mathrm{cl}}=\left(\mathfrak{Z}\left(\mathscr{N}_{0}\right), \Xi,\left(\psi_{\xi}^{\mathrm{cl}}\right)_{\xi \in \Xi}\right)$ be the classical parts of $\mathscr{E}$ and $\mathscr{F}$, respectively. Since we have $\mathscr{E} \otimes \mathscr{F} \sim_{\mathrm{CP}_{\sigma}} \mathscr{E}_{0} \otimes \mathscr{F}_{0}$, Theorem 2 implies that $\mathscr{E}_{0} \otimes \mathscr{F}_{0}$ is the minimal sufficient statistical experiment normally $\mathrm{CP}$ equivalent to $\mathscr{E} \otimes \mathscr{F}$. Therefore

$$
(\mathscr{E} \otimes \mathscr{F})_{\mathrm{cl}} \cong\left(\mathfrak{Z}\left(\mathscr{M}_{0} \bar{\otimes} \mathscr{N}_{0}\right), \Theta \times \Xi,\left(\lambda_{\theta, \xi}\right)_{(\theta, \xi) \in \Theta \times \Xi}\right),
$$

where $\lambda_{\theta, \xi}$ is the restriction of $\varphi_{\theta}^{(0)} \bar{\otimes} \psi_{\xi}^{(0)}$ to the center $\mathfrak{Z}\left(\mathscr{M}_{0} \bar{\otimes} \mathscr{N}_{0}\right)$. On the other hand, it is known that $\mathfrak{Z}\left(\mathscr{M}_{0} \bar{\otimes} \mathscr{N}_{0}\right)=\mathfrak{Z}\left(\mathscr{M}_{0}\right) \bar{\otimes} \mathfrak{Z}\left(\mathscr{N}_{0}\right)$ ([20], Corollary IV.5.11). Thus $\lambda_{\theta, \xi}$ coincides with $\varphi_{\theta}^{\mathrm{cl}} \bar{\otimes} \psi_{\xi}^{\mathrm{cl}}$. Therefore

$$
(\mathscr{E} \otimes \mathscr{F})_{\mathrm{cl}} \cong\left(\mathfrak{Z}\left(\mathscr{M}_{0}\right) \bar{\otimes} \mathfrak{Z}\left(\mathscr{N}_{0}\right), \Theta \times \Xi,\left(\varphi_{\theta}^{\mathrm{cl}} \bar{\otimes} \psi_{\xi}^{\mathrm{cl}}\right)_{(\theta, \xi) \in \Theta \times \Xi}\right)=\mathscr{E}_{\mathrm{cl}} \otimes \mathscr{F}_{\mathrm{cl}},
$$

which completes the proof. 


\section{Concluding remark}

In this paper we have given an operational meaning of the classical part $\mathscr{E}_{\mathrm{cl}}$ of a statistical experiment $\mathscr{E}$. Namely, $\mathscr{E}_{\mathrm{cl}}$ is the accessible part of $\mathscr{E}$ when we do not disturb $\mathscr{E}$. In the formulation of the accessibility relations, we have identified the outcome composite system with the algebraic or a $C^{*}$-tensor product of the outcome algebras. In the von Neumann algebra setup considered in this paper, it is also possible to identify the outcome composite system with the normal tensor product. The accessibility relation defined by the normal tensor product is more restrictive than the accessibility relation $\Subset$ considered in this paper, similarly as the notion of broadcastability presented in [10] is more restrictive than that defined here. Then it is natural to ask how these accessibility relations differ, mathematically and operationally. We leave this as an open question.

\section{A Proofs of Lemmas 2 and 3}

In this appendix, we give direct proofs of Lemmas 2 and 3

Proof of lemma 2 From the universality of $\mathscr{M}^{* *}$, there exists a normal representation $\tilde{\pi}: \mathscr{M}^{* *} \rightarrow \mathscr{M}$ satisfying $\tilde{\pi} \circ \pi_{\mathscr{M}}=\mathrm{id} \mathscr{M}$. Then for each $A \in \mathscr{M}$ and each $\theta \in \Theta$ we have

$$
\overline{\varphi_{\theta}}\left(\pi_{\mathscr{M}}(A)\right)=\varphi_{\theta}(A)=\varphi_{\theta} \circ \tilde{\pi}\left(\pi_{\mathscr{M}}(A)\right) .
$$

Since $\pi \mathscr{M}(\mathscr{M})$ is ultraweakly dense in $\mathscr{M}^{* *}$ and $\overline{\varphi_{\theta}}$ and $\varphi_{\theta} \circ \tilde{\pi}$ are normal, we obtain $\overline{\varphi_{\theta}}=\varphi_{\theta} \circ \tilde{\pi}$ for each $\theta \in \Theta$. Hence $\mathscr{E} \preccurlyeq \mathrm{CP}_{\sigma} \mathscr{E}$. On the other hand, since the kernel ker $\tilde{\pi}$ is a self-adjoint, ultraweakly closed, two-sided ideal on $\mathscr{M}^{* *}$, there exists a central projection $\widetilde{P} \in \mathfrak{Z}\left(\mathscr{M}^{* *}\right)$ such that ker $\widetilde{\pi}=\widetilde{P}^{\perp} \mathscr{M}^{* *}$, where $\widetilde{P}^{\perp}:=\mathbb{1}_{\mathscr{M}^{* *}}-\widetilde{P}$. Then the restriction $\left.\tilde{\pi}\right|_{\widetilde{P} \mathscr{M}^{* *}}$ of $\tilde{\pi}$ to the (possibly non-unital) von Neumann subalgebra $\widetilde{P} \mathscr{M}^{* *}$ is a normal isomorphism from $\widetilde{P} \mathscr{M}^{* *}$ onto $\tilde{\pi}\left(\mathscr{M}^{* *}\right)=\mathscr{M}$. We write the inverse of $\left.\tilde{\pi}\right|_{\widetilde{P} \mathscr{M}^{* *}}$ as $\rho: \mathscr{M} \rightarrow \widetilde{P} \mathscr{M}^{* *}$. We define a normal CP channel $\Phi \in \mathbf{C h}_{\sigma}^{\mathrm{CP}}\left(\mathscr{M} \rightarrow \mathscr{M}^{* *}\right)$ by $\Phi(A):=\rho(A)+\phi_{0}(A) \widetilde{P}^{\perp}$ $(A \in \mathscr{M})$, where $\phi_{0}$ is a fixed normal state on $\mathscr{M}$. Then for each $A \in \mathscr{M}$ and each $\theta \in \Theta$, we have

$$
\overline{\varphi_{\theta}} \circ \Phi(A)=\varphi_{\theta} \circ \tilde{\pi} \circ \Phi(A)=\varphi_{\theta}\left(\tilde{\pi} \circ \rho(A)+\phi_{0}(A) \tilde{\pi}\left(\widetilde{P}^{\perp}\right)\right)=\varphi_{\theta}(A)
$$

which implies $\mathscr{E} \preccurlyeq \mathrm{CP}_{\sigma} \overline{\mathscr{E}}$. Thus we have shown $\mathscr{E} \sim \mathrm{CP}_{\sigma} \overline{\mathscr{E}}$.

Proof of Lemma 3. We have only to prove the implication $\mathscr{E} \preccurlyeq \mathrm{CP} \mathscr{F} \Longrightarrow \mathscr{E} \preccurlyeq \mathrm{CP}_{\sigma} \mathscr{F}$. Assume $\mathscr{E} \preccurlyeq \mathrm{CP} \mathscr{F}$ and take a channel $\Phi \in \mathbf{C h}^{\mathrm{CP}}(\mathscr{M} \rightarrow \mathscr{N})$ such that $\varphi_{\theta}=\psi_{\theta} \circ \Phi$ for all $\theta \in \Theta$. Let $\Phi^{* *}: \mathscr{M}^{* *} \rightarrow \mathscr{N}^{* *}$ be the double dual map of $\Phi$. Then $\Phi^{* *}$ is an extension of $\Phi$ that is continuous in the ultraweak topologies of $\mathscr{M}^{* *}$ and $\mathscr{N}^{* *}$, respectively.

Now we show that $\Phi^{* *}$ is CP. For this, we have to show that for each integer $n \geq 1$ and each $\left\{A_{i}^{\prime \prime}\right\}_{i=1}^{n} \subseteq \mathscr{M}^{* *}$, the $n \times n$ matrix $\left(\Phi^{* *}\left(A_{i}^{\prime \prime *} A_{j}^{\prime \prime}\right)\right)_{i, j=1}^{n}$ is positive. By the Kaplansky density theorem, we can take nets $\left(A_{i \alpha}\right)$ on $\mathscr{M}$ satisfying $\left\|A_{i \alpha}\right\| \leq\left\|A_{i}^{\prime \prime}\right\|$ and $\pi_{\mathscr{M}}\left(A_{i \alpha}\right) \stackrel{u s *}{\longrightarrow} A_{i}^{\prime \prime}(i=1, \ldots, n)$, where $\stackrel{u s *}{\longrightarrow}$ denotes the ultrastrong* convergence on $\mathscr{H}_{\mathscr{M}}$. Then we have $\pi_{\mathscr{M}}\left(A_{i \alpha}^{*} A_{j \alpha}\right) \stackrel{u s *}{\longrightarrow} A_{i}^{\prime \prime *} A_{j}^{\prime \prime}$ and hence $\pi_{\mathscr{N}} \circ \Phi\left(A_{i \alpha}^{*} A_{j \alpha}\right)=$ $\Phi^{* *} \circ \pi_{\mathscr{M}}\left(A_{i \alpha}^{*} A_{j \alpha}\right) \stackrel{u w}{\longrightarrow} \Phi^{* *}\left(A_{i}^{\prime \prime *} A_{j}^{\prime \prime}\right)$, where $\stackrel{u w}{\longrightarrow}$ denotes the ultraweak convergence. Hence the matrix $\left(\Phi^{* *}\left(A_{i}^{\prime \prime *} A_{j}^{\prime \prime}\right)\right)_{i, j=1}^{n}$ is an ultraweak limit of the net of positive matrices $\left(\pi_{\mathscr{N}} \circ \Phi\left(A_{i \alpha}^{*} A_{j \alpha}\right)\right)_{i, j=1}^{n}$, and therefore is also positive. Thus $\Phi^{* *}$ is a normal $\mathrm{CP}$ channel

We denote the normal extensions of $\mathscr{E}$ and $\mathscr{F}$ by $\overline{\mathscr{E}}=\left(\mathscr{M}^{* *}, \Theta,\left(\overline{\varphi_{\theta}}\right)_{\theta \in \Theta}\right)$ and $\overline{\mathscr{F}}=\left(\mathscr{N}^{* *}, \Theta,\left(\overline{\psi_{\theta}}\right)_{\theta \in \Theta}\right)$, respectively. Then for each $A \in \mathscr{M}$ we have

$$
\overline{\psi_{\theta}} \circ \Phi^{* *}\left(\pi_{\mathscr{M}}(A)\right)=\overline{\psi_{\theta}}\left(\pi_{\mathscr{N}}(\Phi(A))\right)=\psi_{\theta} \circ \Phi(A)=\varphi_{\theta}(A)=\overline{\varphi_{\theta}}\left(\pi_{\mathscr{M}}(A)\right) .
$$

Since $\pi_{\mathscr{M}}(\mathscr{M})$ is ultraweakly dense in $\mathscr{M}^{* *}$, this implies $\overline{\varphi_{\theta}}=\overline{\psi_{\theta}} \circ \Phi^{* *}(\theta \in \Theta)$, and hence $\overline{\mathscr{E}} \preccurlyeq \mathrm{CP}_{\sigma} \overline{\mathscr{F}}$. Thus from Lemma 2 we obtain $\mathscr{E} \preccurlyeq \mathrm{CP}_{\sigma} \mathscr{F}$, which completes the proof. 
Acknowledgements The author would like to thank Takayuki Miyadera for helpful discussions. He is also grateful to Erkka Haapasalo for helpful comments on the first version of this paper.

\section{References}

1. Barnum, H., Barrett, J., Leifer, M., Wilce, A.: Generalized no-broadcasting theorem. Phys. Rev. Lett. 99, 240501 (2007). DOI 10.1103/PhysRevLett.99.240501. URL https://link.aps.org/doi/10.1103/PhysRevLett.99.240501

2. Barnum, H., Caves, C.M., Fuchs, C.A., Jozsa, R., Schumacher, B.: Noncommuting mixed states cannot be broadcast. Phys. Rev. Lett. 76, 2818-2821 (1996). DOI 10.1103/PhysRevLett.76.2818. URL https://link.aps.org/doi/10.1103/PhysRevLett.76.2818

3. Brown, N.P., Ozawa, N.: $C^{*}$-algebras and finite-dimensional approximations, vol. 88. American Mathematical Soc. (2008)

4. Chacón, J.E., Montanero, J., Nogales, A.G., Pérez, P.: A note on minimal sufficiency. Stat. Sinica 16(1), 7-14 (2006). URL http://www.jstor.org/stable/24307476

5. Choi, M.D.: A Schwarz inequality for positive linear maps on $C^{*}$-algebras. Illinois J. Math. 18(4), 565-574 (1974). URL http://projecteuclid.org/euclid.ijm/1256051007

6. Dieks, D.: Communication by EPR devices. Phys. Lett. A 92(6), 271 272 (1982). DOI http://dx.doi.org/10.1016/0375-9601(82)90084-6. URL http://www.sciencedirect.com/science/article/pii/0375960182900846

7. Hayden, P., Jozsa, R., Petz, D., Winter, A.: Structure of States Which Satisfy Strong Subadditivity of Quantum Entropy with Equality. Commun. Math. Phys. 246(2), 359-374 (2004). DOI 10.1007/ s00220-004-1049-z. URL http://dx.doi.org/10.1007/s00220-004-1049-z

8. Hiai, F., Mosonyi, M., Petz, D., Bény, C.: Quantum $f$-divergences and error correction. Rev. Math. Phys. 23(07), 691-747 (2011). DOI 10.1142/S0129055X11004412. URL http://www.worldscientific.com/doi/abs/10.1142/S0129055X11004412

9. Jenčová, A., Petz, D.: Sufficiency in quantum statistical inference. Commun. Math. Phys. 263(1), 259-276 (2006). DOI 10.1007/s00220-005-1510-7. URL http://dx.doi.org/10.1007/s00220-005-1510-7

10. Kaniowski, K., Lubnauer, K., Łuczak, A.: Cloning and broadcasting in operator algebras. Quart. J. Math. 66(1), 191 (2015). DOI 10.1093/qmath/hau028. URL http://dx.doi.org/10.1093/qmath/hau028

11. Kaniowski, K., Lubnauer, K., Łuczak, A.: Cloning in $C^{*}$-Algebras. Proc. Edinburgh Math. Soc. 60(3), 689705 (2017). DOI 10.1017/S0013091516000328

12. Koashi, M., Imoto, N.: Operations that do not disturb partially known quantum states. Phys. Rev. A 66, 022318 (2002). DOI 10.1103/PhysRevA.66.022318. URL http://link.aps.org/doi/10.1103/PhysRevA.66.022318

13. Kümmerer, B., Nagel, R.: Mean ergodic semigroups on $W^{*}$-algebras. Acta Sci. Math. 41(1-2), 151159 (1979)

14. Kuramochi, Y.: Minimal sufficient statistical experiments on von neumann algebras. J. Math. Phys. 58(6), 062,203 (2017). DOI 10.1063/1.4986247. URL http://dx.doi.org/10.1063/1.4986247

15. Kuramochi, Y.: Quantum incompatibility of channels with general outcome operator algebras. arXiv preprint arXiv:1708.00150v1 (2017)

16. Lindblad, G.: A general no-cloning theorem. Lett. Math. Phys. 47(2), 189-196 (1999). DOI 10.1023/ A:1007581027660. URL http://dx.doi.org/10.1023/A:1007581027660

17. Łuczak, A.: Quantum sufficiency in the operator algebra framework. Int. J Theor. Phys. 53(10), 3423-3433 (2014). DOI 10.1007/s10773-013-1747-4. URL http://dx.doi.org/10.1007/s10773-013-1747-4

18. Park, J.L.: The concept of transition in quantum mechanics. Found. Phys. 1(1), 23-33 (1970). DOI 10.1007/BF00708652

19. Størmer, E.: On projection maps of von Neumann algebras. Math. Scand. 30(1), 46-50 (1972). URL http://www.jstor.org/stable/24491050

20. Takesaki, M.: Theory of Operator Algebras I. Springer (1979)

21. Thomsen, K.: Invariant states for positive operator semigroups. Stud. Math. 81(3), 287-291 (1985)

22. Umegaki, H.: Conditional expectation in an operator algebra, III. Kodai Math. Sem. Rep. 11(2), 51-64 (1959). DOI 10.2996/kmj/1138844157. URL http://dx.doi.org/10.2996/kmj/1138844157 
23. Umegaki, H.: Conditional expectation in an operator algebra. IV. Entropy and information. Kodai Math. Sem. Rep. 14(2), 59-85 (1962). DOI 10.2996/kmj/1138844604. URL http://dx.doi.org/10.2996/kmj/1138844604

24. Wootters, W.K., Zurek, W.H.: A single quantum cannot be cloned. Nature 299(5886), 802-803 (1982) 